IZA DP No. 4773

Optimal Use of Labor Market Policies:

The Role of Job Search Assistance

Conny Wunsch

February 2010 


\title{
Optimal Use of Labor Market Policies: The Role of Job Search Assistance
}

\author{
Conny Wunsch \\ SEW, University of St. Gallen, \\ CESifo and IZA

\section{Discussion Paper No. 4773 \\ February 2010}

\author{
IZA \\ P.O. Box 7240 \\ 53072 Bonn \\ Germany \\ Phone: +49-228-3894-0 \\ Fax: +49-228-3894-180 \\ E-mail: iza@iza.org
}

\begin{abstract}
Any opinions expressed here are those of the author(s) and not those of IZA. Research published in this series may include views on policy, but the institute itself takes no institutional policy positions.

The Institute for the Study of Labor (IZA) in Bonn is a local and virtual international research center and a place of communication between science, politics and business. IZA is an independent nonprofit organization supported by Deutsche Post Foundation. The center is associated with the University of Bonn and offers a stimulating research environment through its international network, workshops and conferences, data service, project support, research visits and doctoral program. IZA engages in (i) original and internationally competitive research in all fields of labor economics, (ii) development of policy concepts, and (iii) dissemination of research results and concepts to the interested public.
\end{abstract}

IZA Discussion Papers often represent preliminary work and are circulated to encourage discussion. Citation of such a paper should account for its provisional character. A revised version may be available directly from the author. 
IZA Discussion Paper No. 4773

February 2010

\section{ABSTRACT}

\section{Optimal Use of Labor Market Policies: The Role of Job Search Assistance}

This paper studies the role of job search assistance programs in optimal welfare-to-work programs. The analysis is based on a framework, that allows for endogenous choice of benefit types and levels, wage taxes or subsidies, and activation measures such as monitoring and job search assistance for each period of unemployment in a dynamic environment with negative duration dependence in the exit rates to employment and potential depreciation in reemployment wages. We show that the main role of job search assistance is to delay or prevent situations in which it is no longer optimal to incentivize the worker to provide positive search effort. It is used to restore or maintain some minimum exit rate to employment which increases with the cost-effectiveness of job search assistance. We also find that in line with existing policies, these programs should mainly be used at the beginning of unemployment and for short durations. However, contrary to existing schemes, they should be exclusively targeted at unemployed workers with low initial exit rates to employment. For all other workers, they should only be used if they fail to find a job within reasonable time despite high expected initial exit rates.

JEL Classification: J64, J65, J68, D82, D86

Keywords: job search, optimal unemployment insurance, welfare-to-work policies, recursive contracts

Corresponding author:

Conny Wunsch

Swiss Institute for Empirical Economic Research

University of St. Gallen

Varnbüelstrasse 14

9000 St. Gallen

Switzerland

E-mail: conny.wunsch@unisg.ch

\footnotetext{
* I thank Gerard van den Berg, Monika Bütler, Michael Lechner, Alan Manning, Blaise Melly, Fabio Trojani, Stephan Wiehler as well as anonymous referees and various seminar participants for helpful discussions and comments. I also thank Michael Lechner for providing some of his GAUSS procedures, adapted versions of which I used for some of the estimations. The usual disclaimer applies.
} 


\section{Introduction}

In most countries, labor market policies for the unemployed rely on two sets of measures. Passive income support during unemployment provides consumption insurance and gives job seekers the opportunity to look for appropriate jobs. Active measures aim at increasing job seekers' exit rates to employment, e.g. by improving search effectiveness or skills. Welfare-to-work (WTW) programs are government programs that combine different active and passive measures. Expenditures on such programs are substantial, ranging from $1 \%$ to $5 \%$ of GDP in OECD countries (OECD, 2007). Job search assistance programs belong to the most widely used activation measures in OECD countries (OECD, 2007) because they are relatively inexpensive and because they have proven to be quite effective in many countries (e.g. Fay, 1996; Heckman et al., 1999; Martin and Grubb, 2001; Kluve and Schmidt, 2002; Thomsen, 2009).

This paper studies the role of job search assistance programs in optimal welfare-to-work programs. The analysis is based on an adapted version of the framework developed by Pavoni and Violante (2007). This framework allows for endogenous choice of benefit types and levels, wage taxes or subsidies, and activation measures for each period of unemployment in a dynamic environment, which is characterized by negative duration dependence in the exit rates to employment and potential depreciation in reemployment wages. We extend the model by Pavoni and Violante (2007) in two ways. First, we add job search assistance (JA) as a means to raise exit rates to employment to the set of policy measures considered by Pavoni and Violante (2007). These measures include benefits conditional on job search (named unemployment insurance), benefits unconditional on job search (named social assistance), and job search monitoring. Second, we take into account that some worker characteristics like job search skills affect the job finding probability but not productivity in the job. Pavoni and Violante (2007) only consider human capital that is assumed to affect both the exit rates and the reemployment wages.

We show that the optimal policy strongly depends on the job finding probabilities. Therefore, neglecting some important determinants of the exit rates as in Pavoni and Violante (2007) may lead to different optimal decisions. Moreover, we find that introducing JA has important implications for the dynamics of the model: in contrast to Pavoni and Violante (2007), depreciation in reemployment wages or negative duration dependence in the exit rates to employment are no longer necessary for adjustments in the choice of policy instruments to be optimal during unemployment. If JA is 
sufficiently effective and job finding probabilities are low, it may be optimal to use such a program at the beginning of unemployment and switch to passive measures or monitoring after exit rates have increased sufficiently.

Based on a quantitative analysis for an economy that resembles West Germany in the period 20002002, we analyze the optimal use of JA as a function of its ability to raise job finding probabilities. West Germany is an interesting case to study because it is comparable to most industrialized OECD countries and it is the largest among the typical continental European economies, where JA is an important and intensively used instrument to combat unemployment. Moreover, we can use exceptionally rich and large administrative data that allow for most parameters of the model to be estimated semi- or non-parametrically for the same sample of interest, which is a major improvement over most existing calibrations of economic models.

We show that the main role of JA is to delay or prevent situations in which it is no longer optimal to incentivize the worker to provide positive search effort. If sufficiently effective, it is used to restore or maintain some minimum exit rate to employment which increases with the cost-effectiveness of JA. The minimum effectiveness required for JA to be used at all in the optimal policy is at the lower bound of the empirical estimates, implying that JA will usually be part of optimal WTW programs. We also find that in line with existing policies, JA should mainly be used at the beginning of unemployment and for short durations. However, contrary to existing schemes, JA should be exclusively targeted at unemployed workers with low initial exit rates to employment. For all other workers, it should only be used if they fail to find a job within reasonable time despite high expected initial exit rates.

Finally, a comparison of the policy actually implemented in West Germany in the period 20002002 with the optimal scheme indicates a substantial inefficiency of the German system with respect to both benefit payments and the use of JA. Actual long-run benefits were too high and expenditures on JA were wasted as JA was ineffective in this period. We also show that there would have been considerable gains from implementing tight monitoring in West Germany, at least for workers with relatively low initial exit rates to employment.

Most existing studies that take a normative perspective on WTW programs have entirely focused on the optimal time profile of benefits when search effort is unobservable (some of the main contributions are Shavell and Weiss, 1979; Wang and Williamson, 1996; Davidson and Woodbury, 
1997; Hopenhayn and Nicolini, 1997; Cahuc and Lehmann, 2000; Fredriksson and Holmlund, 2001). This literature shows that benefits have to decline with unemployment duration in order to provide search incentives. Most of the still limited normative literature that takes into account both passive and active measures studies the role of job search monitoring. For example, Fredriksson and Holmlund (2006) and Boone et al. (2007) compare optimal unemployment insurance with and without monitoring and sanctions in a general equilibrium framework. Assuming random assignment of monitoring, they show that welfare increases when monitoring is used. In a partial equilibrium framework, Setty (2009) and Wunsch (2009a) endogenize monitoring intensity and the size of the benefit sanction and derive their optimal values as a function of unemployment duration, and in the case of Wunsch (2009a), also of worker characteristics. Other active measures have been analyzed by Coles and Masters (2000), who consider job creation subsidies and retraining, and Blanchard and Tirole (2008), who study layoff taxes. Moreover, Cardullo and Van der Linden (2007) and Van der Linden $(2003 a, b)$ explicitly analyze interactions between the design of the unemployment insurance system and the use of active measures like employment subsidies, monitoring, and training.

The studies that are most closely related to our work are Spinnewijn (2009) and Wunsch (2009b) who analyze the optimal use of training that increases work-related skills in frameworks similar to Pavoni and Violante (2007), i.e. with human capital depreciation during unemployment. They show that training can be used to prevent reemployment wages from deteriorating to levels where providing search incentives is no longer optimal. Combining this result with our results for job search assistance implies complementary roles for training and JA: in the presence of both non-negligible depreciation in reemployment wages and negative duration dependence in exit rates to employment, only the combination of both measures can prevent the returns to search from deteriorating to prohibitively low levels during unemployment. Another related study comes from Ribi (2009), who analyzes the optimal size of job search assistance programs in a static general equilibrium framework with homogeneous agents. She shows that there is a trade off between the positive effects of JA on the participants and the marginal increase in taxes that is needed to finance JA and distorts employment decisions. Moreover, she finds that the optimal size of the program decreases with increasing generosity of the unemployment system as additional funds have to be raised.

The remainder of the paper is organized as follows. The next section describes the details of the model. Section 3 contains the quantitative analysis for West Germany. We describe in detail how the model is parameterized using the administrative data and discuss the results for various 
interesting policy questions. The last section concludes. The appendix contains the proof of the main theoretical result. Additional information on the data is provided in a data appendix that is available on the Internet. ${ }^{1}$

\section{The model}

This paper uses an adapted but also somewhat simplified version of the framework developed by Pavoni and Violante (2007) that focuses on the role of job search skills rather than human capital and productivity in the job. The key elements of the framework by Pavoni and Violante (2007) that are also part of the adapted model are outlined in the next section. Afterwards, the adapted version of the model is discussed in detail.

\subsection{The setup}

Following Hopenhayn and Nicolini (1997), the setup considered by Pavoni and Violante (2007) is a dynamic principal-agent problem. A planner (the principal), e.g. the representative of the unemployment insurance or the welfare system, faces an unemployed worker (the agent) whose effort with respect to his activity is unobservable to the planner. Usually, it is assumed that the worker's only activity is job search. However, as illustrated in Pavoni and Violante (2005), the worker may alternatively participate in, and devote effort to, a WTW program such as training. The objective of the planner is to design a contract which insures the worker against failure of his activity by providing some income during unemployment, and which, at the same time, incentivizes the worker to exert an appropriate level of effort.

At the beginning of unemployment, at time $t=0$, the risk-neutral planner offers the risk-averse agent, the unemployed worker, a contract which insures the worker against failure of job search or participation in JA. This contract maximizes expected discounted net fiscal revenue of the insurer, ${ }^{2}$ subject to providing the agent with at least an expected discounted utility level of $U_{0}$. From a policy perspective, this is a more relevant case than the dual problem of utilitarian expected welfare maximization subject to a budget constraint. Moreover, it allows writing the optimal contract in a convenient recursive form (see Spear and Srivastava, 1987). The promised utility, $U_{0}$, is exogenously given, e.g. the outcome of voting, and can be regarded as a measure of the generosity of the welfare

\footnotetext{
1 Http://www.alexandria.unisg.ch/publications/Conny_Wunsch/58068.

2 Note that this is equivalent to minimizing net expenditure.
} 
system. Its level may depend on worker characteristics. In particular, the planner can give different weights to different types of individuals in the social welfare function by requiring different $U_{0}$. Net fiscal revenue is tax revenue if the worker is employed, and expenditures on benefit payments, monitoring, and JA if the worker is unemployed.

Workers are infinitely lived and maximize expected discounted lifetime utility. They have timeseparable preferences over consumption, $c \geq 0$, and effort, $a$. Agents discount the future at rate $\beta \in(0,1)$, and period utility is given by $u\left(c_{t}\right)-a_{t}$. Effort is assumed to be either high $(a=e>0)$ or low $(a=0)$, which underlines the role of fixed costs and the extensive margin of participation decisions. However, the disutility of effort during employment is generally not restricted to be the same as during unemployment, ${ }^{3}$ but it must be ensured that accepting a job offer always dominates staying unemployed.

The planner can observe whether the worker is employed or unemployed, his activity (job search or participation in JA) during unemployment, and the outcome of the worker's activity, $y \in\{s, f\}$, where $s$ denotes success and $f$ failure. But the planner cannot observe the worker's effort choice, $a$, so that he faces a moral hazard problem.

For each period $t$ and contingent on all observable histories up to $t$, the contract specifies the transfers to the worker, the policy instrument to be used, and the corresponding recommended effort choice of the worker.

For simplification, it is assumed that workers do not have access to savings, insurance or credit markets. In particular, it is assumed that workers cannot self-insure against the random outcome $y \in\{s, f\}$ of their activity, e.g. by saving. Pavoni and Violante (2005) show that when workers can save through credit markets but still face a no-borrowing constraint, which is a reasonable assumption for unemployed workers, the same optimal contract can be implemented by introducing a linear, time-invariant interest tax.

\subsection{Introducing job search assistance}

One important limitation of the model by Pavoni and Violante (2007) is that it only allows for active measures that affect both the job finding rate and the reemployment wages (through an increase in human capital). Although this covers both formal training and programs that provide work

\footnotetext{
3 For example, assuming that effort is the same during employment and search would imply that the worker searches for a job with the same intensity and time input as he works.
} 
experience, it excludes JA programs, which are one of the most widely used activation measures for the unemployed in OECD countries (OECD, 2007). The objective of these programs is to help those unemployed without any severe skill deficits to find a job as quickly as possible. JA programs offer a combination of intensified counseling and training to improve job search skills, e.g. by teaching the participants how to locate job vacancies and how to fill out job applications, or by helping them to prepare for job interviews. But they may also include some availability checks or other forms of weak monitoring that make jobseekers search more effectively. Therefore, they affect the employment hazard but not productivity in the job. In particular, they do not affect wages. Usually, these programs are short and thus less costly than traditional training or wage subsidies.

Besides being relatively inexpensive, the key argument in favor of JA programs is that they have proven to be quite effective in many countries, especially those programs with a substantial amount of job application training (for evidence, see in particular the recent survey by Thomsen, 2009). ${ }^{4}$ This explains both the popularity and importance of JA programs. Therefore, these programs should be taken into account when considering the optimal design of labor market policies. For this reason, the economy considered by Pavoni and Violante (2007) is enriched to allow for JA programs.

To study the role of JA in optimal WTW programs, individual heterogeneity in job search skills is introduced into the model. A new state variable, $p$, is added that subsumes all individual factors that affect the exit rate from unemployment to employment under the label of search effectiveness. In order to both keep the model tractable and to focus on the implications of heterogeneity in search effectiveness and the role of job search assistance, the theoretical analysis abstracts from heterogeneity in, and depreciation of, reemployment wages. However, in the quantitative analysis we will consider this case as well..$^{5}$

In our model, the job finding probability of an unemployed worker depends on search effectiveness, $p$, and search effort, $a \in\{0, e\}$. The corresponding hazard rate from unemployment to employment is denoted by $\pi(p, a)$, and it is assumed that $\pi(p, 0) \equiv 0$ and that $\pi(p, e) \equiv \pi(p) \in(0,1)$ is continuous and increasing in $p$. We also assume that there is negative duration dependence in $\pi$, which is an empirically well established fact in labor economics (see, e.g., the survey of the relevant literature by Machin and Manning, 1999). In the model, negative duration dependence is induced by a depreciation in search effectiveness which can be motivated by the stock-flow matching approach

\footnotetext{
4 For more evidence, see Fay (1996); Heckman et al. (1999); Martin and Grubb (2001); Dolton and O’Neill (2002); Kluve and Schmidt (2002); Blundell et al. (2004); OECD (2005).

${ }^{5}$ See also the theoretical analysis presented in an earlier version of this paper (Wunsch, 2007).
} 
(Coles and Smith, 1998). At the beginning of unemployment, job seekers start with the existing stock of vacancies and may look for a particular type of job. If their job search activities fail, they will have exhausted this stock of vacancies after some time and will start sampling from a new flow of vacancies. They may also have to change or extend the range of jobs they are looking for.

Job search assistance makes it possible to increase initially low levels of search effectiveness or to restore search skills that depreciate with increasing unemployment duration. It is introduced as a technology that increases search effectiveness with probability $\theta(a)$. This probability depends on the effort exerted during program participation, where $\theta \equiv \theta(e)>\theta(0) \equiv 0$. For simplification, to avoid the necessity of modeling time allocation within a period, it is assumed that search and program participation are mutually exclusive activities within a period, implying that participants cannot exit to employment directly from the program. ${ }^{6}$ However, since the length of a period can be arbitrarily small, this assumption is not restrictive.

\subsection{The planner's problem}

The optimization problem of the planner is formulated in the recursive form proposed by Pavoni and Violante (2007), with job search assistance added to the set of policy instruments and search effectiveness as state variable. Human capital as state variable ( $h$ in Pavoni and Violante, 2007) is suppressed because, for simplification and focus as already mentioned above, the theoretical analysis abstracts from ex-ante heterogeneity in, and depreciation of, human capital. Therefore, a state is defined by the promised continuation utility, $U$, and search effectiveness, $p$, i.e. by the pair $(U, p)$. At the beginning of each period, the planner chooses the optimal policy instrument, $i(U, p)$, for an unemployed worker who enters the period with state $(U, p)$, by solving

$$
V(U, p)=\max _{i \in\{J A, J M, S A, U I\}} V^{i}(U, p),
$$

where $V$ is the upper envelope of the values associated with the different policies, which are described in detail below. In each case, the planner chooses an effort recommendation $a(U, p) \in\{0, e\}$, the transfer $c(U, p)$ and the continuation utilities $U^{y}(U, p)$ conditional on the outcome $y \in\{f, s\}$ of the worker's activity. There is always a promise-keeping (PK) constraint, which ensures delivery of the

\footnotetext{
6 This corresponds to a so-called lock-in effect of program participation, which has been documented in many empirical studies (e.g. Gerfin and Lechner, 2002; Van Ours, 2004; Lechner et al., 2010; Sianesi, 2004, 2007; Jespersen et al., 2008).
} 
promised utility $U$ to the worker. Moreover, whenever positive effort is required and effort cannot be verified, there is also an incentive compatibility (IC) constraint which ensures that it is optimal for the worker to choose this effort level.

Following Hopenhayn and Nicolini (1997), employment is assumed to be absorbing without informational asymmetries in order to concentrate on the (current) unemployment experience, ${ }^{7}$ and the planner can impose a wage tax or pay a wage subsidy as additional policy instruments. For an employed worker and state $(U, p)$ the planner solves

$$
\begin{array}{r}
W(U)=\max _{c, U^{s}} w-c+\beta W\left(U^{s}\right) \\
\text { s.t. } \quad U=u(c)-e^{W}+\beta U^{s},
\end{array}
$$

where $e^{W}$ denotes the disutility of work. $W$ is independent of $p$ because employment is absorbing.

Under the unemployment insurance (UI) scheme, the worker is required to search with effort $e$, and finds employment with probability $\pi(p)$. With probability $1-\pi(p)$, the worker remains unemployed, and job search skills depreciate to a level $p^{f} \leq p$. The planner's problem is given by

$$
\begin{aligned}
V^{U I}(U, p)= & \max _{c, U^{f}, U^{s}}-c+\beta\left[\pi(p) W\left(U^{s}\right)+(1-\pi(p)) V\left(U^{f}, p^{f}\right)\right] \\
\text { s.t. } & U=u(c)-e+\beta\left[\pi(p) U^{s}+(1-\pi(p)) U^{f}\right] \\
& U \geq u(c)+\beta U^{f} .
\end{aligned}
$$

The first and the second constraints are the promise-keeping (PK) and the incentive compatibility (IC) constraints, respectively. Using the PK constraint, the cost of having to obey the IC constraint can be expressed as $U_{U I}^{s}-U_{U I}^{f} \geq \frac{e}{\beta \pi(p)}$. It is increasing in the required effort level and decreasing in the employment hazard which, in turn, is increasing in $p$. Hence, this cost increases as search effectiveness depreciates during UI. Note that besides the incentive cost, there is also an effort compensation cost.

Under the social assistance ( $S A)$ scheme, the worker is not required to search $(a=0)$, and receives some transfer that ensures delivery of promised utility $U$. Since effort is zero, no IC constraint is needed, which implies that no incentive or effort compensation costs have to be incurred. The

\footnotetext{
7 Qualitative results for the same unemployment spell do not change as long as the job separation rate is exogenous. Optimal contracts with endogenous job separation are studied by Zhao (2000) and Hopenhayn and Nicolini (2009), who show that in this case the optimal contract has to take into account the worker's full employment history.
} 
planner's problem is given by

$$
\begin{gathered}
V^{S A}(U, p)=\max _{c, U^{f}, U^{s}}-c+\beta V\left(U^{f}, p^{f}\right) \\
\text { s.t. } \quad U=u(c)+\beta U^{f} .
\end{gathered}
$$

If the planner chooses to monitor the search activities of the agent by using job search monitoring $(J M)$, he is able to verify the worker's search effort against payment of a cost $\kappa^{J M}$ and solves

$$
\begin{gathered}
V^{J M}(U, p)=\max _{c, U^{f}, U^{s}}-c-\kappa^{J M}+\beta\left[\pi(p) W\left(U^{s}\right)+(1-\pi(p)) V\left(U^{f}, p^{f}\right)\right] \\
\text { s.t. } \quad U=u(c)-e+\beta\left[\pi(p) U^{s}+(1-\pi(p)) U^{f}\right] .
\end{gathered}
$$

Note that the planner will never combine monitoring with low effort because any deviation from no search can be observed at no additional cost since $\pi(p, 0)=0$. Also note the absence of the IC constraint because here, the planner can verify search effort so that there is no incentive problem during JM. This implies that monitoring is assumed to be perfect. Although monitoring may be imperfect in reality, this serves as an interesting benchmark case for the optimal design of labor market policies. The absence of the IC constraint implies zero incentive costs, but the effort compensation cost remains as high effort is required from the worker.

Job search assistance $(J A)$ is introduced in a similar way as training in Pavoni and Violante (2005). If the worker is enrolled in JA, the planner solves

$$
\begin{aligned}
V^{J A}(U, p)= & \max _{c, U^{f}, U^{s}}-c-\kappa^{J A}+\beta\left[\theta V\left(U^{s}, p^{s}\right)+(1-\theta) V\left(U^{f}, p^{f}\right)\right] \\
\text { s.t. } & U=u(c)-e+\beta\left[\theta U^{s}+(1-\theta) U^{f}\right] \\
& U \geq u(c)+\beta U^{f} .
\end{aligned}
$$

Job search assistance is costly, requiring payment of a fixed cost, $\kappa^{J A}$. It raises search effectiveness with probability $\theta(a) .{ }^{8}$ Since it is assumed that $\theta(0)=0$, the program will always be combined with high effort, as the cost of the program would not be compensated by any return if effort were zero. The assumption that search and program participation are mutually exclusive activities within a period can be seen from the value function in case of success of program participation. It is not the

\footnotetext{
8 This incorporates the idea that the (expected) program effect is increasing in effort.
} 
value of employment, $W\left(U^{s}\right)$, but the value of continued unemployment of the worker, $V\left(U^{s}, p^{s}\right)$, with improved search skills $p^{s} \geq p$. Since positive effort is required $(a=e>0)$, both the PK and the IC constraints are needed, implying that both the incentive and the effort compensation costs have to be incurred..$^{9}$ Note that in contrast to UI, the incentive costs, which can be characterized as $U_{i}^{s}-U_{i}^{f} \geq \frac{e}{\beta \theta}$, remain constant during JA while they increase during UI because of the negative duration dependence in $\pi(p)$.

\subsection{Optimal sequence of transfers and policies}

With constant reemployment wages, the structure of the model with search skills becomes very similar to the one of Pavoni and Violante (2007) with human capital. In fact, in terms of the dynamics, the model is an intermediate case between the two extremes studied by Pavoni and Violante (2007), namely no dynamics, where both the exit rate and the reemployment wages are constant during unemployment, and the case where both fall. As a consequence, most of the results proven by Pavoni and Violante (2007) continue to hold in the adapted model presented here. ${ }^{10}$ They are summarized in the following paragraphs.

Because of the absence of the IC constraint, under employment and JM (no information asymmetry) as well as under SA (no effort required) it is easy to see that promised utility remains constant and full insurance will be provided. Consequently, benefits remain constant during JM and SA. Moreover, since employment is absorbing, this implies that the value of an employed worker simplifies to $W(U)=\frac{w-c^{W}}{1-\beta}$, where $c^{W}(U)=u^{-1}\left((1-\beta) U+e^{W}\right)$. The implicit wage tax (if positive) or subsidy (if negative) that is imposed on employed workers is thus given by $\tau(U)=w-c^{W}(U)$ and constant during employment. In the cases of UI and JA, the presence of the IC constraint requires promised utility and hence benefits to fall upon failure of job search or JA (as punishment) and to increase upon their success (as reward). ${ }^{11}$

The wage tax/subsidy upon reemployment is one instrument to incentivize the worker. Because promised utility remains constant during JM, the wage tax/subsidy upon reemployment remains

\footnotetext{
${ }^{9}$ Note that monitoring the worker's effort in the program is very difficult because the worker's learning effort, in particular his attention and concentration, are very hard to verify.

10 The same is true in a model that allows for ex-ante heterogeneity in and depreciation of both human capital (wages) and search effectiveness For details see the first version of this paper (Wunsch, 2007).

11 This result underlines the generality of the result first derived by Shavell and Weiss (1979). Note that this is actually in line with empirical studies on the effect of sanctions, which suggest that reductions in benefits do seem to succeed in restoring incentives to search or to participate in activation measures (Van den Berg et al., 2004; Abbring et al., 2005; Lalive et al., 2005).
} 
constant as well as long as reemployment wages are unchanged. On the other hand, the wage tax (subsidy) increases (decreases) during UI and JA because promised utility falls if search effectiveness remains constant. Once it depreciates, the opposite may happen.

Regarding the optimal sequence of policies, Pavoni and Violante (2007) show that SA is always absorbing because it is used at a point where it is no longer optimal to incentivize the worker and where search skills continue to deteriorate, which further decreases the returns to search. They also show that if both reemployment wages and the exit rates to employment remain constant during unemployment, UI and JM are absorbing as well because no policy change is required if the returns to search do not change.

When search effectiveness depreciates during unemployment, neither UI nor JM are absorbing. Since search effectiveness behaves in the same way during JM and UI, the main difference between JM and UI is that the incentive cost incurred during UI is replaced by the fixed monitoring cost. Since the incentive cost increases because of depreciation of $p$ while the monitoring cost remains fixed, UI will only be used before JM as long as the incentive cost is lower than the fixed cost, $\kappa^{J M}$. Moreover, depreciation of $p$ implies that a switch to JM becomes more likely with increasing unemployment duration. Once $\kappa^{J M}$ is lower than the incentive cost during UI, a switch back to UI may only occur after successful use of JA because otherwise, the incentive cost keeps rising for UI. Essentially, JM is a policy instrument to save incentive costs in the presence of negative duration dependence in the exit rate to employment. Consequently, the typical sequence of policies in the absence of JA is UI followed by JM and then by SA.

Having summarized the main results derived by Pavoni and Violante (2007), let us now look at the role of JA in the model. JA has three main cost components. The first one is absent under UI and JM. It is the fact that participants cannot exit to employment directly form JA. However, since the length of a period can be arbitrarily small this is not a big issue. The second cost component is the incentive cost which is absent under JM but not under UI. Yet, the incentive cost under UI, $U^{s}-U^{f} \geq \frac{e}{\beta \pi(p)}$, increases with the unemployment duration, while the incentive cost of JA, $U^{s}-U^{f} \geq \frac{e}{\beta \theta}$, remains constant. Thus, the use of JA relative to UI becomes more likely the longer the worker remains unemployed. Finally, there is the fixed cost, $\kappa^{J A}$, which is absent under UI but, although potentially with a different amount, present under JM. Consequently, we have the following situation: the cost of UI is increasing over time, while those of JA and JM are constant. 
JA will be preferred to UI once the incentive cost of UI is higher than the incentive cost of JA plus the fixed cost of JA. The advantage of JM relative to JA is the absence of the incentive cost and a potentially smaller fixed cost. However, in contrast to JA, JM cannot prevent the deterioration of search effectiveness and thus the use of SA. Because the worker no longer searches for a job under SA and because SA is absorbing, the optimality of SA implies extremely high net expenditures in the long run. In the absence of JA, JM is used to save costs when the the deterioration of search skills and the use of SA cannot be avoided. Once JA is available, it can be used to delay or even prevent the use of SA, which creates a potential for considerably larger cost savings than with the use of JM. Consequently, JA is likely to crowd out JM.

The possibility to use JA has another very important implication for the dynamics of the model. One of the most important results shown by Pavoni and Violante (2007) is that human capital dynamics, i.e. the deterioration of reemployment wages or the exit rate to employment, are necessary for policy transitions to occur because the returns to search remain constant in this case. With the availability of JA in a setting with constant reemployment wages, this is no longer the case, even without depreciation in search effectiveness. The reason is that even though UI, JM, and SA are still absorbing in this case, JA might be optimal at the beginning of unemployment. This is particularly likely if at the initial values of the state variables, $\left(U_{0}, p_{0}\right)$, the job finding probability, $\pi\left(p_{0}\right)$, is very low and the returns to JA are large because the success probability of JA, $\theta$, is high. When JA is used at the beginning of unemployment for low levels of $p_{0}$, search effectiveness will eventually increase. At some point, $p$ will be sufficiently large that a switch to UI or potentially JM will be optimal, which will then remain optimal for the rest of the unemployment spell as $p$ does not decrease. The following proposition, which is proven in the appendix, states the conditions under which deterioration of the job finding probability is still necessary for policy transitions to occur in a model with JA:

Proposition: If reemployment wages are constant and search effectiveness does not fall during unemployment, the following result holds: if $J A$ is not optimal at $(U, p)$ and $\theta \leq \pi(p)$, JA will never be optimal thereafter if $V$ is submodular.

Because all other policies are absorbing if reemployment wages are constant and search effectiveness does not deteriorate, this proposition implies that if JA is not used in period 0, i.e. at $\left(U_{0}, p_{0}\right)$, it will never be used if $\theta \leq \pi\left(p_{0}\right)$. Because of the latter condition, this is more likely the larger the 
initial search effectiveness, $p_{0}$, and the lower the success rate of JA, $\theta$. However, whenever JA is optimal at $\left(U_{0}, p_{0}\right)$, the optimal policy will eventually switch to JM and UI because the returns to search and the incentive costs during UI change when using JA. In the next section it will be shown that usually, JA is indeed used under the optimal policy. Thus, the presence of job search assistance in the model implies much richer dynamics than the setup studied by Pavoni and Violante (2007).

\section{Quantitative analysis}

In order to derive more detailed insights into the optimal choice over policy instruments, the model is parameterized to resemble the West German economy in the period 2000-2002. West Germany is an interesting case to study because it is comparable to most industrialized OECD countries and it is the largest among the typical continental European economies, where WTW programs are important and intensively used instruments to combat unemployment. Moreover, in the course of substantial reforms of the German unemployment insurance system, there have been heated debates about its optimal design in recent years.

\subsection{The West German policy scheme 2000-2002}

Before discussing how the model is parameterized, the West German policy scheme of the period 2000-2002 is briefly described. In Germany, unemployment insurance is mandatory and employees who have contributed for at least 12 months within the 3 years before entering unemployment are eligible for unemployment benefits (UB) if they register with the public employment service (PES). The minimum UB entitlement is 6 months. In the period under consideration, the maximum claim increased stepwise with the total contribution time in the 7 years before becoming unemployed, as well as age, up to a maximum of 32 months at age 54 or above with previous contributions of at least 64 months. Since 1994, the replacement rate is $67 \%(60 \%)$ of previous average net earnings from insured employment with (without) dependent children.

Until 2005, unemployed workers could become eligible for unemployment assistance (UA) after exhaustion of UB. In contrast to UB, UA was means tested and potentially indefinite. However, like UB, UA was proportional to previous earnings but with lower replacement rates than UB (57\% and $53 \%$ with and without dependent children, respectively). Those unemployed who were ineligible for UB and UA could receive social assistance, which was a fixed monthly payment unrelated to previous 
earnings, means-tested, and administered by local authorities. In 2005, one of the largest reforms of the German unemployment insurance system became effective. The maximum UB entitlement was cut to 12 and 18 months for unemployed workers below and above age 55, respectively. Moreover, UA and social assistance were combined to a new fixed means-tested welfare payment of normally 345 EUR, which is called unemployment benefits II. ${ }^{12}$

Actual payment of benefits is conditional on active job search, regular show-up at the PES, and participation in labor market programs. In case of noncompliance with benefit conditions, sanctions, i.e. reductions in or suspensions of benefits, can be imposed. However, in the period under consideration, search activities were not monitored very strictly due to capacity constraints within the PES.

With respect to WTW programs, job search assistance has become by far the most important activation measure in terms of the number of participants (1.1 million during the period 20002002). Expenditures are moderate (on average, about 570 EUR per participant) because durations are short (up to three months but usually about 1.5 months). In Germany, job search assistance combines some counseling with a substantial training component as well as some availability checks. The training component focuses on how to locate job vacancies, how to fill out job applications, and how to prepare for job interviews. The courses are usually full-time, which fits very well to how job search assistance is modeled in the theoretical part. ${ }^{13}$

\subsection{Data and population of interest}

With the exception of the preference and program cost parameters, all parameters of the model are estimated using a large administrative database which has been built up by the German Institute for Employment Research. This approach has several advantages compared to the usual procedure of picking values from different studies that are based on different data, samples, time periods and potentially even different countries. First, it ensures that all parameters represent the same population in a specific time period and a specific economic and institutional environment. Second, it is much more transparent as to how the estimates are generated, making it much easier to judge their validity. Third, it allows linking the simulation results to the population observed in the data, which considerably improves the interpretation and understanding of the results.

\footnotetext{
12 In addition, UB II recipients can receive remuneration for housing costs (including water, electricity, and heating).

13 For further details on the German unemployment insurance system and WTW programs, see Wunsch (2006).
} 
The database used is a $2 \%$ random sample from all individuals who have been subject to German social insurance since 1990. It covers the period 1990-2005 and combines spell information from social insurance records, program participation records, and the benefit payment and job seeker registers of the PES. The database comprises very detailed and accurate information on several dimensions. It provides detailed information on employment status on a daily basis for the period 1990-2005. Personal characteristics include education, age, gender, marital status, number of children, profession, nationality, disabilities, and health. The benefit payment register provides information on type and amount of benefits received, remaining benefit claims, and imposition of sanctions. The job seeker register includes information on the desired form of employment, compliance with benefit conditions, and the number of placement propositions by the PES. Moreover, the data comprise information on job characteristics such as type of employment, industry, occupation, qualification, and wages. With respect to program participation, the data cover the type of the program as well as the planned and actual durations. Detailed regional information, which includes federal state, local unemployment rate, migration, demographic and industry structure, infrastructure and urbanity, complements the database (see the data appendix A on the Internet for a full list of variables).

For the simulation, the model is parameterized to resemble the population of West German workers who entered unemployment from employment between January 2000 and December 2002, and who received benefits from the unemployment insurance system (UB or UA). ${ }^{14}$ To concentrate on the main body of the workforce, apprentices, young men on civilian or military service, as well as elderly workers in special forms of employment are excluded. This reduced sample consists of 76,304 observations and is referred to as the reference population of interest (RPI) in the remainder of the paper.

\subsection{Parameterizing the model}

\subsubsection{State variables, wages, monitoring cost and preferences}

Time units are defined by half-months. This is the smallest level of aggregation which is reasonable with the data. Initial promised utility $U_{0} \equiv U\left(p_{0}\right)$ delivered to a worker with characteristics $p_{0}$ is set equal to the value implied by the actual system. It is obtained by plugging in the policy parameters,

\footnotetext{
14 This excludes recipients of social assistance, which is administered by local authorities for which no common register exists. For this reason, individual data on these persons are unavailable in Germany.
} 
and solving backwards for the promised utility levels. The necessary information, i.e. the amount of benefits and the fraction of the RPI subject to each policy per half-month of unemployment are calculated directly from the data. In the numerical solution, $U$ is treated as a continuous variable but is evaluated at 500 equidistant points in the interval $[50,1000]$.

Reemployment wages are set to the level of the wage from the employment spell before entering unemployment. ${ }^{15}$ For most of the analysis, the median wage per half-month in the RPI is used (860 EUR per half-month or 1720 EUR per month). To analyze how changes in reemployment wages affect the results, the median wages in the upper (1340 EUR per half-month or 2680 EUR per month) and lower third (440 EUR per half-month or 880 EUR per month) of the wage distribution are considered as well.

A ranking of the individuals in terms of search effectiveness is obtained by estimating a probit model for the probability of exiting unemployment within three months of becoming unemployed as a function of personal characteristics (age, gender, education, nationality, number and age of kids, marital status, health), detailed information on ten years of individual employment histories (fraction employed/unemployed/out of labor force/in programs, number and duration of past (un)employment spells, employment status in specific periods), characteristics of the last job (wage, full/part-time, industry, occupation, qualification), remaining UI claim, amount of benefit, compliance with benefit conditions, regional characteristics, as well as year and quarter dummies. The predicted probabilities for each individual yield the distribution of $p$ in the RPI (see the data appendix $\mathrm{B}$ on the Internet for the exact specification of the probit model, the estimated coefficients and the distribution of predicted probabilities). In the numerical solution, $p$ is discretized to 30 grid points. For each grid of $p$, the value functions for all policies with respect to $U$ are computed using Chebychev polynomials up to the 20 th order.

To parameterize the cost of tight monitoring, the average gross salary of a caseworker per halfmonth (about 1200 EUR according to BA, 2001-2005) is divided by the number of unemployed workers a caseworker can reasonably take care of in a half-month. A conservative number would be 20, which yields a value of 60 EUR. Allowing for some administrative cost, the baseline value for $\kappa^{J M}$ is set to 100 EUR.

Preferences are parameterized as follows. The half-monthly discount factor $\beta$ is chosen to match

$\overline{15}$ For part-time workers the wage is scaled up to obtain an approximate fulltime equivalent. 
an interest rate of $4 \%$ per annum, which prevailed in the EURO area in the period of interest. Period utility over consumption is assumed to be logarithmic, i.e. $u(c) \equiv \ln (c)$, but specifications with intertemporal elasticities of substitution below and above one are tested as well. To calibrate the disutility of effort, $e$, an approach that originates from common practice in calibrating macroeconomic models is used. Let the disutility of time spent working, $n$, be logarithmic as well, and denote by $\phi$ the relative weight on leisure versus consumption. Assuming a standard Cobb-Douglas production function, the static optimality condition of the worker yields a value of $\phi=2.35$ given a labor share of 0.73 (BMAS, 2003), a consumption-income ratio of 0.72 (Statistisches Bundesamt, 2000-2002), and a fraction of time spent working of $n=0.3$. This implies a value for the disutility of work effort of $e=\phi[\ln (1)-\ln (1-n)]=0.84$ (Chari et al., 1995). For the baseline calibration, it is assumed that the disutility of effort during unemployment equals the one during work. Yet, as a sensitivity check, the case where only half of the time spent on work is spent on search and program participation is considered as well.

\subsubsection{Exit rates to employment}

In the numerical solution, $p$ is discretized to 30 grid points. The job finding probability corresponding to the largest grid value of $p$ is calibrated to the probability of exiting to employment within the first month ${ }^{16}$ of unemployment, which is estimated non-parametrically from the individuals in the highest five percentiles of the distribution of $p$ in the sample, yielding $\pi_{\max }=19 \%$. The exit rates corresponding to the other grid points for $p$ are geometrically spaced at rate $10 \%$ between $\pi_{\text {max }}$ and $\pi_{\min }=0 .{ }^{17}$ The corresponding exit rates as a function of the descretized values of $p$ are displayed in panel (a) of Figure 1.

The depreciation rate of search effectiveness is estimated semi-parametrically as follows: we are interested in the dependence of the exit rate to employment on unemployment duration for the RPI if nobody participates in any activation measure. Ideally, we would like to observe the exit probability of everyone in the RPI for different unemployment durations. However, a substantial part of the RPI participates in some program which potentially affects the exit rate, and we observe the exit rates between time $t$ and $t+1$ only for the subpopulation of the RPI that is still unemployed at $t$. The problem is that program participation is highly selective and that unemployment duration is

\footnotetext{
16 Because of labor market frictions, the exit rate is very low in the first half-month, and so the one-month exit rate is a better proxy.

17 The results are insensitive to whether zero or a small positive number is chosen for $\pi_{m i n}$.
} 
Figure 1: Exit rates to employment as a function of $p$ and unemployment duration
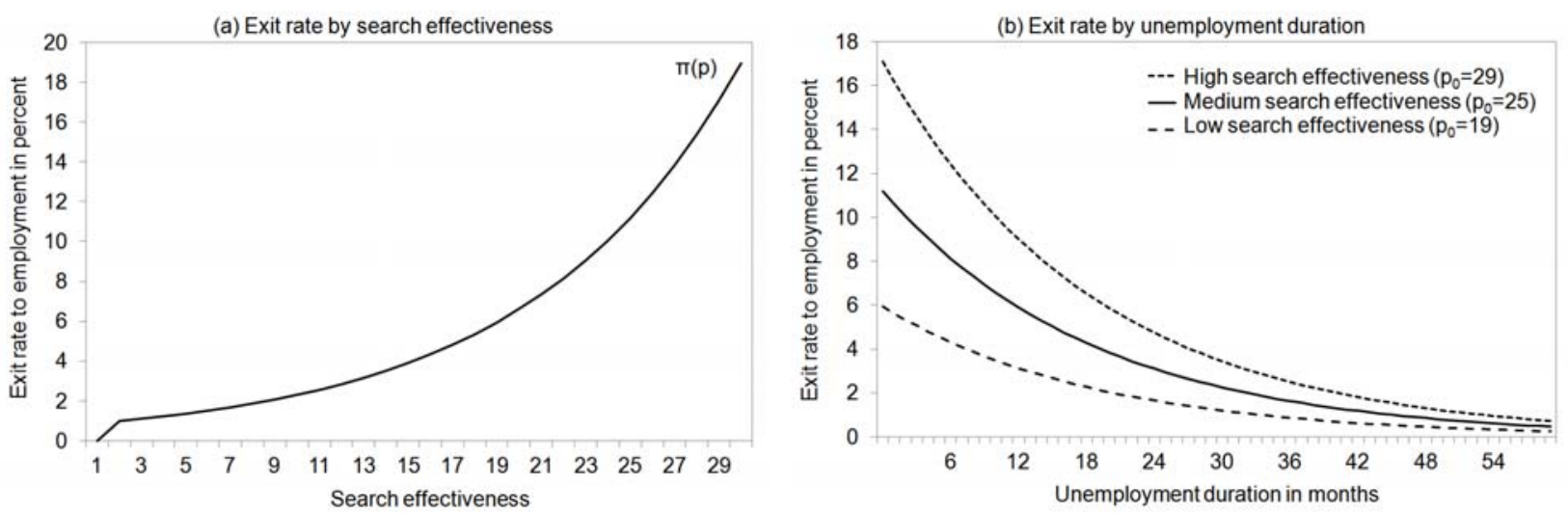

Note: High/medium/low search effectiveness in panel (b) refers to the mean exit rate in the first month of unemployment in the upper/middle/lower third of the distribution of $p$ in the sample.

endogenous: program participants and non-participants differ systematically in characteristics that also affect the exit rate, and the same holds for individuals with different unemployment durations.

To correct for these differences, all individuals that remain unemployed for at least $x$ months and do not participate in any program are matched to the RPI with respect to all characteristics that affect the exit rate and cause selection bias. Matching, i.e. selection based on observables, is justified because the data allow us to observe all key factors that determine the job finding probability. It particular, we not only observe the standard set of socio-demographic characteristics but also health/disability, the kind of job that a person is looking for, occupation and industryspecific experience, incentives provided by the UI system in terms of benefit claims and benefit sanctions, compliance with benefit conditions as a proxy for job search motivation and intensity, and past performance on the labor market over the last 10 years, which allows controlling indirectly for ability, motivation, productivity, and individual employment-related preferences. Finally, we are also able to capture differences in regional labor market performance in a detailed way.

The estimator used is a robust radius matching estimator with regression adjustment proposed by Lechner et al. (2010) and generalized by Lechner and Wunsch (2009), where matching is performed on the basis of the predicted selection probability that is estimated from a probit model, and that uses a triangular kernel to weigh observations. Using this estimator, the unobserved counterfactual average probability of the RPI to find employment within one half-month after $x$ months of unemployment is estimated and compared to the average initial observed exit rate. The estimation is performed separately for $x \in\{3,6,9,12,15,18,21,24,27\}$ months. It turns out that the deprecia- 
tion of the exit rate is of the form $p_{t+1}=(1-\delta) p_{t}$, where $\delta=0.026$ per half-month (see the data appendix $\mathrm{C}$ on the Internet for the exact specification and estimation results of the different probit models and the estimated exit rates as a function of the unemployment duration, as well as data appendix E for the matching protocol). The resulting dependence of the exit rate to employment on unemployment duration is displayed in panel (b) of Figure 1 for different levels of initial search effectiveness.

The depreciation of $p$ is implemented as being stochastic. The respective depreciation probability is calculated from the parameterized depreciation rate $(0.026)$ and the geometric rate at which the grids $p$ are spaced (0.1) which yield a value of 0.26 .

\subsubsection{Job search assistance}

The optimal use of JA will be studied in two ways. First, JA will be calibrated to the actual program implemented in West Germany in the period 2000-2002. This requires estimating the effects of JA from the data. Second, to obtain more general results, the optimal use of JA as a function of the effectiveness of JA is analyzed. For this purpose, the effect on $p$ is fixed to an increase by one grid point, but the success probability, $\theta$, is varied. The fixed cost of JA is calculated from total expenditures per year (excluding benefit payments), the number of participants, and the average program durations from official statistics (BA, 2001-2005). This yields program costs of $\kappa^{J A}=190$ per half-month in EUR.

In order to calibrate the job-search-assistance technology to the German data, estimates of the effects of JA of different durations on the job finding probability for the RPI are required. Each period, the planner has to decide whether the worker should be assigned to a program or, if he already attends a program, whether he should stay for another period. However, when trying to estimate these effects, two kinds of selection problems arise. First, as can be seen from the data appendix A available on the Internet, the participants in JA differ systematically from the RPI in ways that are also related to the job finding probability. Second, actual program durations are potentially endogenous. In the RPI, actual durations differ from planned durations by more than $15 \%$ in $11 \%$ of the cases. Thus, actual program durations cannot be regarded as exogenous.

Given sufficiently rich data, both selection problems can be solved using a so-called dynamic (or sequential) treatment evaluation approach as suggested by Lechner and Miquel (2010). The idea is 
to consider a $\tau$-period program as a sequence of $\tau$ one-period programs and to control for selection at the beginning of each of the $\tau$ periods. Selectivity between program participants and the RPI can then be controlled for at the beginning of the sequence (at $t=0$ ), whereas endogeneity of program durations can be accounted for by selection correction in the $\tau-1$ following periods. The effect of a program of length $\tau$ relative to a program of length $\tau-1$ can then be obtained by comparing the sequence 'participating up to $t=\tau$ ' with the sequence 'participating up to $t=\tau-1$ and not participating in $t=\tau^{\prime} .18$

Here, the population for which the effects are to be estimated is an entry sample into unemployment that is defined independently of any program participation later in the unemployment spell. Lechner and Miquel (2010) show that in this case, the effects of interest are identified under the so-called weak dynamic conditional independence assumption (W-DCIA). This assumption states that, first, conditional on confounding variables at $t=0$, potential outcomes measured from time $\tau \geq 1$ onwards are independent of program participation in period $t=1$. This would account for any selectivity between program participants and the RPI. Second, conditional on participation status up to time $t$ and confounding variables of all periods up to time $t$, potential outcomes are independent of participation in period $t+1$. This would account for endogeneity of program durations. The third part of the W-DCIA is a common-support requirement which demands overlap in the control variables between the populations involved in each of the selection steps.

To judge whether W-DCIA is plausible in this particular application, the confounding variables for program participation relative to the RPI have to be identified. This comparison is mainly driven by the difference between participants in JA and nonparticipants. In addition, those variables that jointly influence the job finding probability and the changes in treatment status (i.e. the decision between staying in JA for another period and leaving) have to be detected.

Selection into programs from the RPI is driven by program eligibility, selection by caseworkers, and self-selection by the unemployed. By construction of the sample, all unemployed workers are eligible because they receive unemployment insurance payments. Caseworkers select on the basis of an assessment of the employment prospects and the specific qualification needs of the unemployed. According to the German legislation, they also have to take into account the chances of successful

\footnotetext{
18 An alternative way of estimating the effect of programs of different durations would be to apply the methodology of Hirano and Imbens (2004) for continuous treatments (see, e.g., Flores-Lagunes et al., 2007). However, this method assumes that selection into programs of different durations is fully determined at the beginning of the program. That is, different program durations are not the outcome of intermediate decisions after the program started.
} 
completion of the program, and the local labor market conditions. Similar arguments apply to self-selection by the unemployed because they also compare their employment prospects with and without a program, as well as the corresponding costs in terms of effort or potentially foregone benefits in case of refusal to participate. Thus, selection is basically driven by the same factors as the ones affecting the exit rate to employment discussed in detail above.

Decisions to leave or stay in the program are driven by factors that change after entering a program. The most important factors are probably the arrival of job offers, exhaustion of benefit claims, and significant changes in health conditions. Other factors may be noncompliance with benefit conditions, changes in family status, moving to another place, and take up or loss of a minor Job, ${ }^{19}$, which, including exhaustion of benefits, are directly observed in the data. Changes in health conditions are observed if they are severe enough to affect unemployment insurance status. The arrival of job offers is not directly observed in the data, but the number of placement propositions by the PES per spell is. ${ }^{20}$ To approximate the arrival of job offers at or up to a specific point in time, a Heckman (1979)-type selection model for the log number of placement propositions per day, that accounts for zero propositions in the spell, is estimated in the subsample of nonparticipants using a rich set of time-invariant (or deterministically changing) and time-varying variables measured at the beginning of the program as explanatory variables. The number of placement propositions at different points in time is then predicted for all individuals in the sample using the updated measurement of the time-varying covariates.

In summary, most of the potentially confounding factors are directly observed in the data. Moreover, those that are not directly observed either can be controlled for indirectly by information on past employment histories and compliance with benefit conditions (ability, motivation, preferences), or they can be approximated by use of observed variables, like arrival of job offers. Thus, the data are sufficiently rich to capture the main sources of selection bias at different points in time before and during program participation. In fact, this is the first application of a dynamic treatment evaluation approach for estimating the effects of programs of different (endogenous) durations. ${ }^{21}$

Lechner (2009) proposes a sequential nearest-neighbor matching estimator where the matching is based on propensity scores to estimate the effects of different sequences of programs for a population

\footnotetext{
19 To provide additional work incentives, benefit recipients can earn additional labor income without losing their claim if they work less than 15 hours per week.

20 A placement proposition is a job vacancy proposed to the job seeker by the caseworker.

21 Another recent paper that uses this approach but focuses on the effects of sequences of different programs, and the timing of program participation is Lechner and Wiehler (2010).
} 
defined within one of the sequences. Consider the case where the interest lies in estimating the effect of the sequence $S^{0}=\left(S_{1}^{0}, S_{2}^{0}\right)$ compared to $S^{1}=\left(S_{1}^{1}, S_{2}^{1}\right)$ for the population defined by $S_{1}^{0}$. In the first step, the population defined by $S_{1}^{1}$ is matched to the population of interest, $S_{1}^{0}$, based on the estimated propensity score of the corresponding selection equation. Then, the population defined by $S_{2}^{1}$ is matched to those observations in $S_{1}^{1}$ that served as matches in the previous step based on the propensity scores from this and the first selection step. In a similar vein, the population defined by $S_{2}^{0}$ is matched to the one defined by $S_{1}^{0}$ based on the corresponding propensity score. To obtain an estimate of the effect of interest, the reweighed outcome of the population defined by $S_{2}^{0}$ is then subtracted from the reweighed outcome of the population defined by $S_{2}^{1}$.

Here, a modified version of this estimator is used. First, the effects are estimated for a population defined outside the sequences under consideration. This implies that an additional matching step has to be performed in each comparison. The populations defined by the first element of each sequence under consideration have to be matched to the RPI before matching within each of the sequences is performed (for the exact specification of the selection models in each step and the corresponding results see the data appendix D on the Internet). ${ }^{22}$ Second, radius matching as proposed by Lechner et al. (2010) for static evaluation problems rather than nearest-neighbor matching is used to increase efficiency and potentially robustness given that the RPI is potentially large compared to the populations defined by the last element of the sequences under consideration. ${ }^{23}$

Table 1 displays the estimated effects of JA of different durations on the probability of finding employment within three months starting from the point in time were treatment status is defined (e.g. after one month of JA when interest is in the effect of completing two versus only one month of JA). The effects are estimated separately for the entire RPI as well as for subsamples defined by the lower, middle, and upper third of the distribution of $p$ in the RPI. It turns out that in West Germany in the period 2000-2002, JA is essentially ineffective independently of program duration. ${ }^{24}$ There seems to be some gain in employment from participating in the program for more than tree rather than only three half-months, but the first half-month of participation seems to have negative impacts. Since program participation is costly, it can be presumed that under these particular

\footnotetext{
${ }^{22}$ See Lechner and Wunsch (2009) for the basic idea of estimating effects for populations defined independently of treatment status.

${ }^{23}$ If the comparison population is small relative to the population for which the effects are estimated, using more than one similar observation prevents that one particular observation gets too much weight.

24 This is consistent with the findings of Wunsch and Lechner (2008), who analyze the effectiveness of different forms of job search assistance using the same database but a static evaluation approach.
} 
circumstances, JA would not be part of the optimal policy.

Table 1: Estimated effects of JA of different durations

\begin{tabular}{l|ccc|ccc|ccc|ccc}
\hline & \multicolumn{3}{|c|}{ Full sample } & \multicolumn{3}{c|}{ Low search } & \multicolumn{3}{c|}{$\begin{array}{c}\text { Medium search } \\
\text { effectiveness }\end{array}$} & \multicolumn{4}{c}{$\begin{array}{c}\text { High search } \\
\text { effectiveness }\end{array}$} & \multicolumn{2}{c}{ effiveness } \\
Comparison & Effect & SE & Obs. & Effect & SE & Obs. & Effect & SE & Obs. & Effect & SE & Obs. \\
\hline JA1 - NP & -0.036 & 0.019 & 1759 & 0.003 & 0.025 & 660 & -0.028 & 0.028 & 673 & $\mathbf{- 0 . 0 9 5}$ & 0.045 & 426 \\
JA2 - JA1 & -0.021 & 0.029 & 1218 & 0.011 & 0.036 & 465 & -0.027 & 0.041 & 454 & 0.044 & 0.066 & 299 \\
JA3 - JA2 & -0.019 & 0.031 & 1182 & -0.040 & 0.035 & 476 & 0.040 & 0.043 & 448 & -0.085 & 0.072 & 258 \\
JA3P - JA3 & $\mathbf{0 . 0 7 9}$ & 0.030 & 1591 & 0.060 & 0.033 & 678 & 0.009 & 0.045 & 569 & 0.062 & 0.079 & 344 \\
\hline
\end{tabular}

Note: Difference in the probability to exit to employment within 3 months in percentage points. NP: nonparticipation, JAn: completion of $n$ half-months of JA. Bold/bold italics/italics indicate significance on the $1 / 5 / 10 \%$ level. Obs. is the number of treated observations. SE is the standard error. For each comparison, less than $1 \%$ of the RPI are deleted due a lack of common support.

\subsection{Results}

\subsubsection{Optimal choice of policies as a function of $(U, p)$}

Before looking at specific policy questions, we want to obtain a better understanding of the dynamics of the model as calibrated to the German economy in the period 2000-2002. Figure 2 displays the optimal choice of policies in the $(U, p)$ space for a medium-skilled worker $(w=1720$ EUR per month) when different choice sets are available. As can be seen from panel (a), if only SA or UI are available, absorbing SA is used when the returns to search, i.e. the job finding probability, $\pi(p)$, are too low to incentivize the worker, or when promised utility is too high, making it too costly to incentivize the worker when returns to search are not sufficiently large. Panel (b) shows that if JM becomes available, it is an effective tool to save the incentive costs of UI when the returns to search are low or promised utility is high, and to prevent SA at very high levels of $U$. At an intermediate value of initial promised utility and high initial search effectiveness, the optimal policy will start with UI. During UI, promised utility falls and search effectiveness deteriorates so that we move to the south west in panel (b), making JM the optimal policy after some time. At low initial levels of $p$, JM will be optimal from the beginning of unemployment. Eventually, $p$ will be so low that the sum of the effort compensation cost and the monitoring cost becomes too high given the very low returns to search, making SA less costly than JM.

In panels (c)-(l), the choice set also includes JA. In these figures, the probability that the job finding probability increases by one grid point (10\%) is varied in 10\%-steps between $10 \%$ and $100 \%$ $(\theta \in\{0.1,0.2, \ldots, 1.0\})$. It turns out that JA is not optimal for any combination $(U, p)$ when $\theta=0.1$.

In fact, the success probability must be at least $15 \%$ for JA to be used at all in the optimal policy 
Figure 2: Optimal choice of policies as a function of $(U, p)$
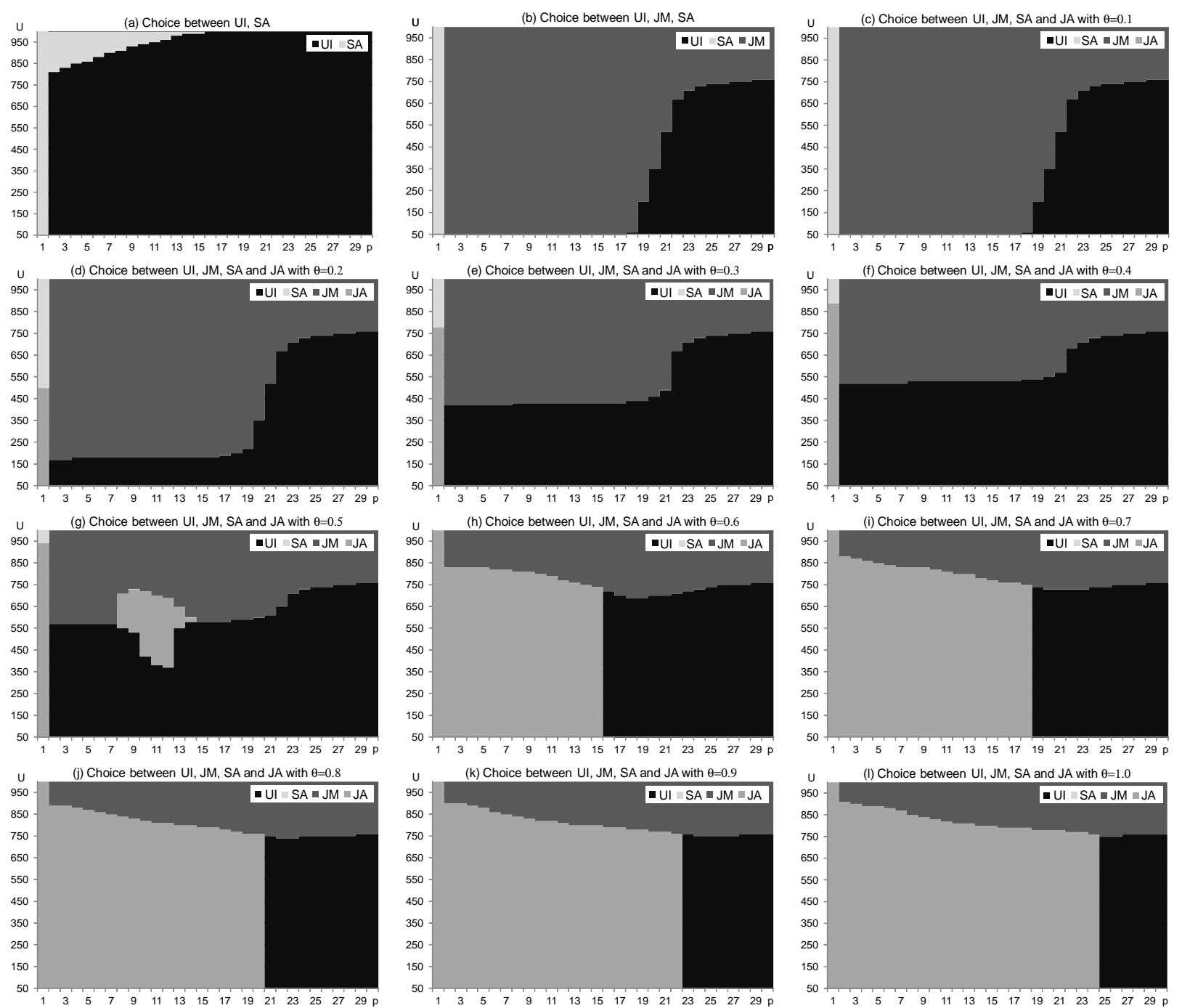

Note: At the right boundary of $p$ where the optimal policy switches from JA to UI, $V^{J A}$ and $V^{U I}$ are very close. Therefore, it can sometimes happen that there are multiple switches between JA and UI as $U$ increases for a given $p$. In such cases, the figures show UI up to the largest value of $U$ for which UI is optimal.

scheme. Clearly, costly JA is not used whenever $\theta \leq \pi(p)$ because in this case the incentive cost exceeds that of UI. However, even if $\theta>\pi(p)$, which is the case for $p \leq 27$ if $\theta=0.15$, the fixed costs of JA prevents its use if the returns to JA are not sufficiently high.

Up to a success probability of $40 \%$, JA will only be used if the returns to search are very low and if promised utility is not too large. If $p$, and hence $\pi(p)$, is very low, the returns to JA are particularly high as not only the returns to search can be increased but also the use of very costly and absorbing SA can be prevented. As a consequence of optimal use of JA at $p=1$, the range of values of $(U, p)$ for which UI is optimal rather than JM increases, thus reducing the importance of JM. The reason is that at any given $(U, p)$, the optimal policy takes into account which policies 
will be optimal in the future once $p$ depreciates or increases after use of JA and as $U$ potentially changes starting from $(U, p)$. As $p$ depreciates during unemployment and if promised utility is not too high, there will eventually be a point in time where JA will be used rather than SA. Since JA is much less costly than SA and since it increases $p$, the planner can afford to use UI also for lower levels of $p$ than in the absence of JA, as long as $U$ is not too high. What happens is that the same utility can be provided for a given level of $p$ by shifting expenditures from later periods, where SA would be optimal in the absence of JA, to earlier periods.

As the success probability of JA increases, the use of JA also increases, crowding out in particular JM but also UI. At $\theta=0.5$, JA will be used not only for $p=1$ but also at intermediate values of $(U, p)$. For lower $U$, UI is still less costly as the fixed cost of JA can be saved. For lower $p$, the prospect to use JA when $p=1$ suffices to keep UI least costly. For $\theta \geq 0.6$, JA is used for all levels of $p$ up to a threshold which increases with $\theta$. Moreover, it is used for all but very high levels of $U$, where the corresponding threshold also increases with $\theta$. Finally note that for $\theta \geq 0.6$, JA is able to prevent the job finding probability from deteriorating to levels at which it is no longer optimal to incentivize the worker. Consequently, very costly SA is no longer part of the optimal policy scheme which implies a potential for considerable budget savings.

In the following sections, the parameterized model is used to answer a range of interesting policy questions. First, the optimality of the policy implemented in West Germany in the period 2000-2002 is assessed by comparing it with the optimal scheme that results when the same initial utility is delivered to workers as implied by the actual system. Second, the potential benefits of tightening monitoring in West Germany are evaluated. Third, to draw some general conclusions on the optimal use of job search assistance, ranges of parameter values for which these programs are optimally used, as well as their optimal duration and timing within the unemployment spell, are derived.

\subsubsection{Assessing the West German policy 2000-2002}

Panel (a) of Figure 3 shows the sequence of policies that would have been optimal for West Germany in the period 2000-2002 in the absence of JM, given that the same level of initial utility $U\left(p_{0}\right)$ is provided as under the actual policy. JM, which is assumed to be perfect in the model, is excluded for the moment because in the period under consideration, search activities were not monitored very strictly due to capacity constraints within the PES. As JA was ineffective in this period, UI and SA are the only policy instruments left. 
As initial promised utility under the actual policy, $U\left(p_{0}\right)$, varies between $430-570$, it can be seen from panel (a) of Figure 2 that SA will only be used at $p=1$, where the returns to search are essentially zero. ${ }^{25}$ Consequently, for $p_{0}>1$, the optimal policy will start with UI but will switch to SA once search effectiveness deteriorates to $p=1$. The higher the initial search effectiveness, $p_{0}$, the longer it will take in the unemployment spell to reach this point. For low $(p=19)$, medium $(p=25)$, and high search effectiveness $(p=29)$, as defined by the mean initial exit rate in the lower, middle, and upper third of the distribution of $p$ in the data, UI will be optimal for unemployment durations of up to $39.5,49$, and 55 months, respectively. It is important to note, though, that this does not mean that maximum unemployment insurance claim durations should be that long. It just means that it is optimal to condition benefit payments on job search effort for this period, regardless of whether they are unemployment insurance payments or welfare payments financed by tax revenue. In the model, UI is just a label for benefits conditional on job search and it has nothing to do with the source of the funding.

Figure 3: Optimal policy for West Germany 2000-2002
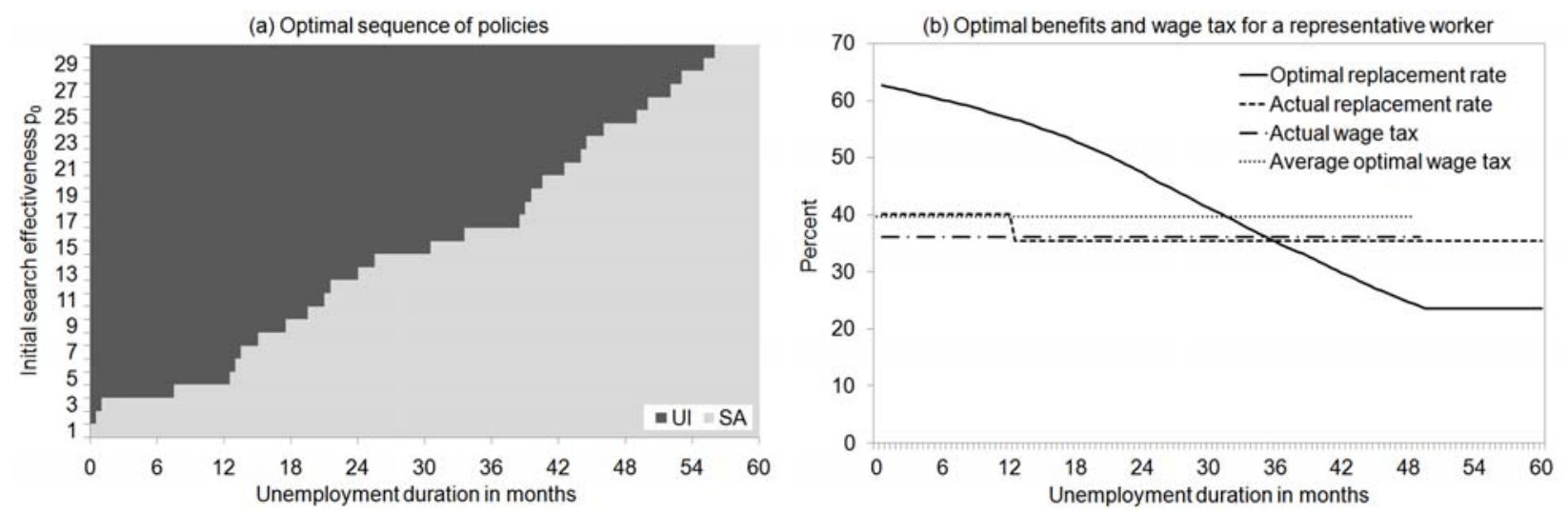

Note: The results displayed in panel (b) are for a worker with median reemployment wage and medium initial search effectiveness $\left(w=1720\right.$ EUR per month, $\left.p_{0}=25, \pi(25)=11 \%\right)$.

For a worker with medium reemployment wage and search effectiveness, panel (b) of Figure 3 shows that optimal benefits decline during UI as predicted by the model to provide intertemporal search incentives by punishing job search failure. The optimal replacement rate starts at $63 \%$ and declines to $24 \%$ when SA becomes optimal. From this point onwards, the benefit remains constant at a level of about 400 EUR per month. Interestingly, this is very close to the level of unemployment benefits II (345 EUR), which have been introduced recently in Germany as the baseline welfare payment that can be received exhaustion of UI.

25 As already stated earlier, the results are insensitive to whether zero or a small positive number is chosen for $\pi(1)$. 
Within the first 36 month of unemployment, optimal benefits are higher than the actual ones, but are considerably smaller thereafter. Lower long-run benefits are the main reason for considerable budget savings that could be realized by implementing the optimal instead of the actual policy. For low, medium, and high initial search effectiveness, the total budget savings amount to 18\%, $4 \%$, and $1 \%$, respectively. The savings are largest for low levels of $p_{0}$ because at low job finding probabilities, expected tax revenue is very low. Consequently, in these cases the amount of long-run benefits, which are too high under the actual policy, is the main determinant of net fiscal revenue. In contrast, for high levels of $p_{0}$ and $\pi\left(p_{0}\right)$, tax revenue has a much larger weight than the savings in terms of long-run benefits under the optimal policy. In fact, for medium search effectiveness in panel (b) of Figure 3, the average optimal wage tax is somewhat larger than the actual one. ${ }^{26}$ This yields higher tax revenue in addition to the budget savings from lower long-run benefits as well as the savings from not using ineffective but costly JA. However, initial benefits are also relatively large, which yields the moderate amount of budget savings of $4 \%$ at $p=25$.

\subsubsection{The value of tight monitoring}

Panel (a) of Figure 4 shows that if monitoring could be tightened to resemble perfect JM, it would be used in the optimal scheme. The total duration for which it is optimal to incentivize agents remains unchanged, because it is determined by the deterioration of the job finding probability, which is unaffected by JM. However, the relative use of JM and UI depends on the relation of the incentive costs during UI to the monitoring cost. If $p$ and $\pi(p)$ are already low at the beginning of unemployment $(p \leq 21, \pi \leq 7 \%)$, the incentive costs of UI are higher than the fixed monitoring cost, making JM optimal from the beginning of unemployment. For sufficiently high levels of $p$ and $\pi(p)$, the optimal policy starts with UI but switches to JM as $\pi(p)$ decreases, and hence the incentive cost of UI increases relative to the fixed monitoring cost and eventually becomes larger than $\kappa^{J M}$. As a consequence, UI is used for longer periods while JM is used for shorter periods the higher $p$. The additional budget savings from JM relative to the optimal policy when only UI and SA are available are very small for high levels of $p$, but can be substantial for low levels of $p$ because in this case, the savings in terms of UI incentive costs are particularly high. For low, medium, and high initial search effectiveness the budget savings are 10\%, $2 \%$, and less than $1 \%$, respectively.

\footnotetext{
26 The optimal wage tax falls as $p$ decreases because a larger utility spread $U^{s}-U^{f} \geq \frac{e}{\beta \pi(p)}$ is needed to incentivize the worker. However, as depreciation of $p$ is implemented stochastically in the simulation, the wage tax will increase as long as $p$ remains constant but promised utility and hence benefits fall during UI.
} 
Figure 4: Optimal use of job search monitoring
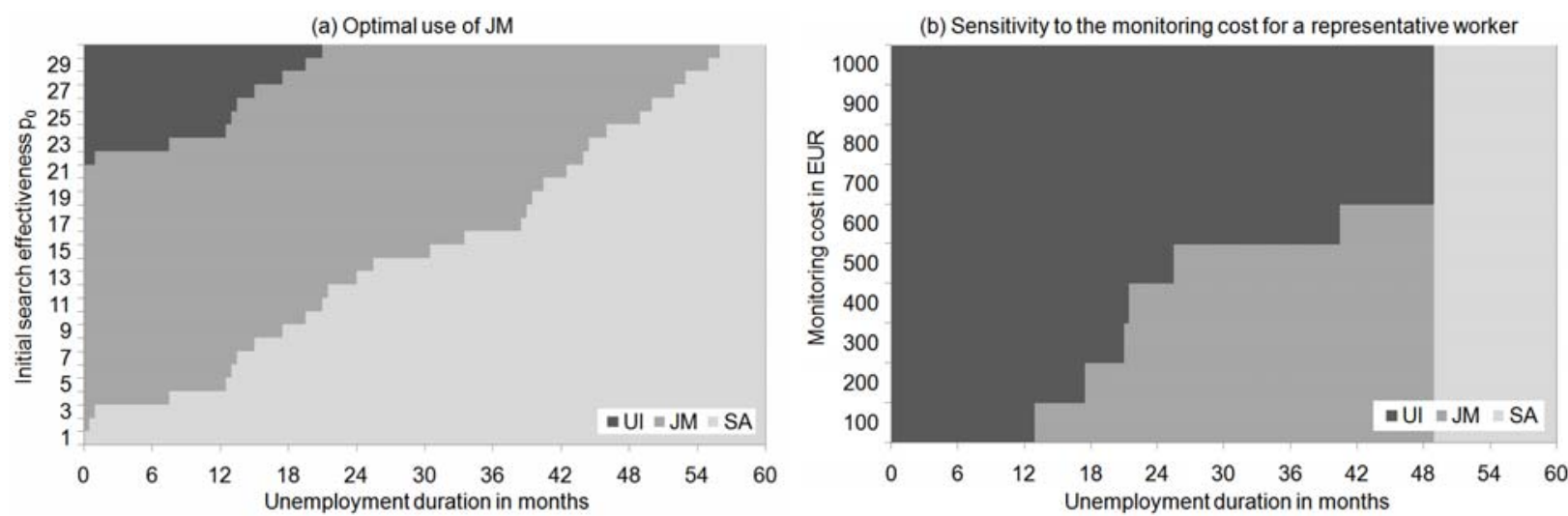

Note: The results displayed in panel (b) are for a worker with median reemployment wage and medium initial search effectiveness $\left(w=1720\right.$ EUR per month, $\left.p_{0}=25, \pi(25)=11 \%\right)$.

In panel (b) of Figure 4, $\kappa^{J M}$ is varied from 100 to 1000 EUR per half-month. The results are displayed for a worker with medium search effectiveness $(p=25)$. Naturally, the use of JM is reduced with increasing cost. JM is used later in the unemployment spell after prolonged use of UI, because the increasing incentive cost of UI stays below the monitoring cost for longer periods. As a result of longer UI, benefits decline to lower levels the larger $\kappa^{J M}$, which compensates to some extent for the higher monitoring cost so that JM is still optimal at some point in the unemployment spell. For $\kappa^{J M} \geq 700$ EUR though, the monitoring cost becomes prohibitively large, making JM no longer optimal.

\subsubsection{Optimal use of job search assistance}

One conclusion from the international evidence on the effects of job search assistance on the job finding probability is that the effectiveness of JA varies with the particular type of the program as well as the time period and country considered. In West Germany, for example, JA has been shown to be ineffective, whereas for both earlier and later periods, positive effects have been estimated (see Thomsen, 2009, for a survey of the German and the international evidence). Therefore, it is important to analyze the optimal use of JA as a function of its ability to raise exit rates to employment. For this purpose, the potential increase in $p$ caused by JA is fixed to one grid point (10\%), and the success probability, $\theta$, is varied in $10 \%$-steps between $10 \%$ and $100 \%$. The corresponding expected causal effects of JA on $\pi(p)$ are displayed in Figure 5. As shown in the note to this figure, the expected potential outcomes, $E\left(\pi_{J A}^{1}\right)$ and $E\left(\pi_{J A}^{0}\right)$, can be calculated from the rate at which the grids for $p$ are spaced $(\gamma=0.1)$, the depreciation probability for $p(\delta=0.26)$, and the success 
probability of JA $(\theta)$. Because a geometric grid is chosen for $p$, the effect in percent is constant across $p$, but increases with $p$ in percentage points. The implied effects in percentage points, which are the usual measures we see in empirical studies, cover the typical range of empirical findings (Fay, 1996; Heckman et al., 1999; Martin and Grubb, 2001; Dolton and O'Neill, 2002; Kluve and Schmidt, 2002; Blundell et al., 2004; OECD, 2005; Thomsen, 2009).

Figure 5: Simulated effect of JA on the exit rate to employment
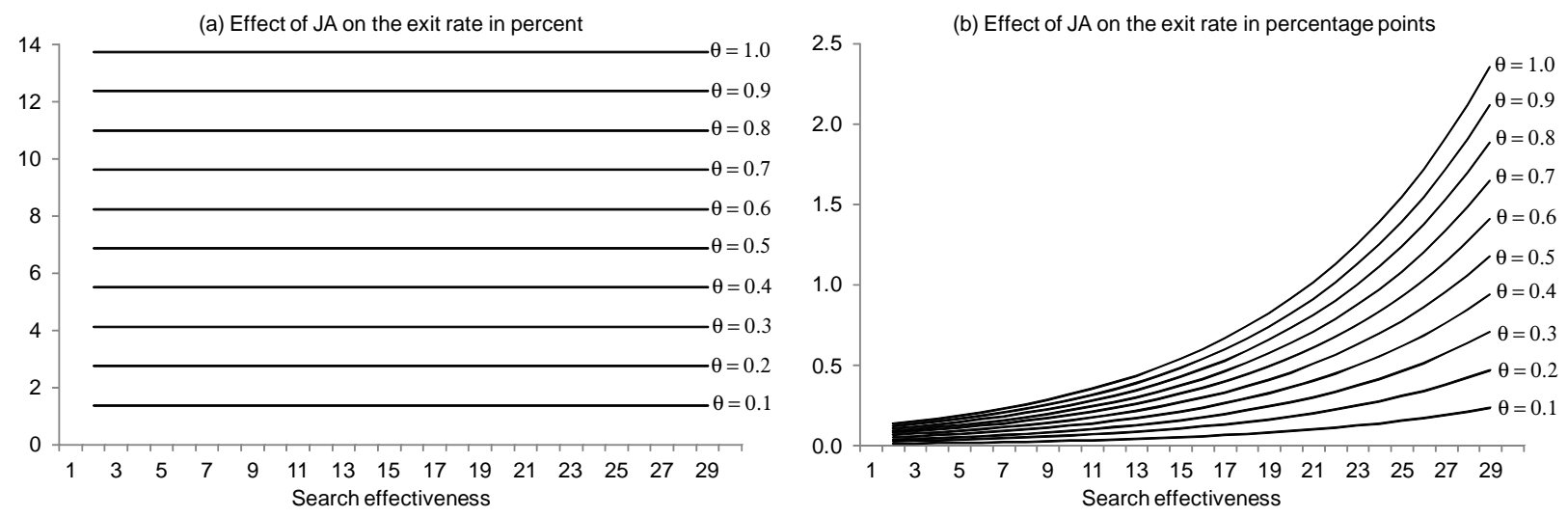

Note: $\quad$ The expected potential outcomes are $E\left(\pi_{J A}^{0}\right)=\delta \pi(p-1)+(1-\delta) \pi(p)=[1-\delta \gamma] \pi(p)$ and $E\left(\pi_{J A}^{1}\right)=$ $\theta \pi(p+1)+(1-\theta) E\left(\pi_{J A}^{0}\right)=\gamma[1 /(1-\gamma)+\delta] \pi(p)$. Panel $(\mathrm{a}):\left[E\left(\pi_{J A}^{1}\right)-E\left(\pi_{J A}^{0}\right)\right] / \pi(p)$. Panel (b): $E\left(\pi_{J A}^{1}\right)-E\left(\pi_{J A}^{0}\right)$.

The first result that was already stated in Section 3.4.1 is that the success probability must be at least $15 \%$ for JA to be optimal at all for any $(U, p)$. For an initial promised utility as implied by the actual West German policy during the period 2000-2002, the required minimum effectiveness is even higher. In this case, $\theta$ must be at least $22 \%$, which corresponds to an expected effect on the exit rate of about $3 \%$ or 0.03 to 0.5 percentage points. However, this is at the lower bound of the empirical estimates, implying that JA will usually be part of optimal WTW programs.

Figure 6 summarizes the main results for the optimal use of JA as a function of $\theta$. Panel (a) displays the range of values of $p$ and the corresponding job finding probabilities, $\pi(p)$, for which it is optimal to use JA. As discussed above, JA is not optimal for $\theta<0.22$. For $0.22 \leq \theta<0.5$, JA should only be used once the exit rate has deteriorated to extremely low levels $(p=1)$ to prevent particularly costly SA. Otherwise, the returns of JA are too small given the still relatively high incentive cost and the fixed cost of JA. At $\theta=0.5$, JA will also be used at intermediate levels of $p$ as discussed in Section 3.4.1. For $\theta \geq 0.6$, JA is optimal for the complete lower range of search effectiveness. This range increases with $\theta$, starting at $p=15$ for $\theta=0.6$ and reaching $p=24$ for $\theta=1$. Note, however, that even for a success probability of one JA is only optimal for the lower 
part of the distribution of search effectiveness or job finding rates: the middle of the distribution lies at $p=25$ or $\pi(25)=11 \%$.

Figure 6: Optimal use of job search assistance
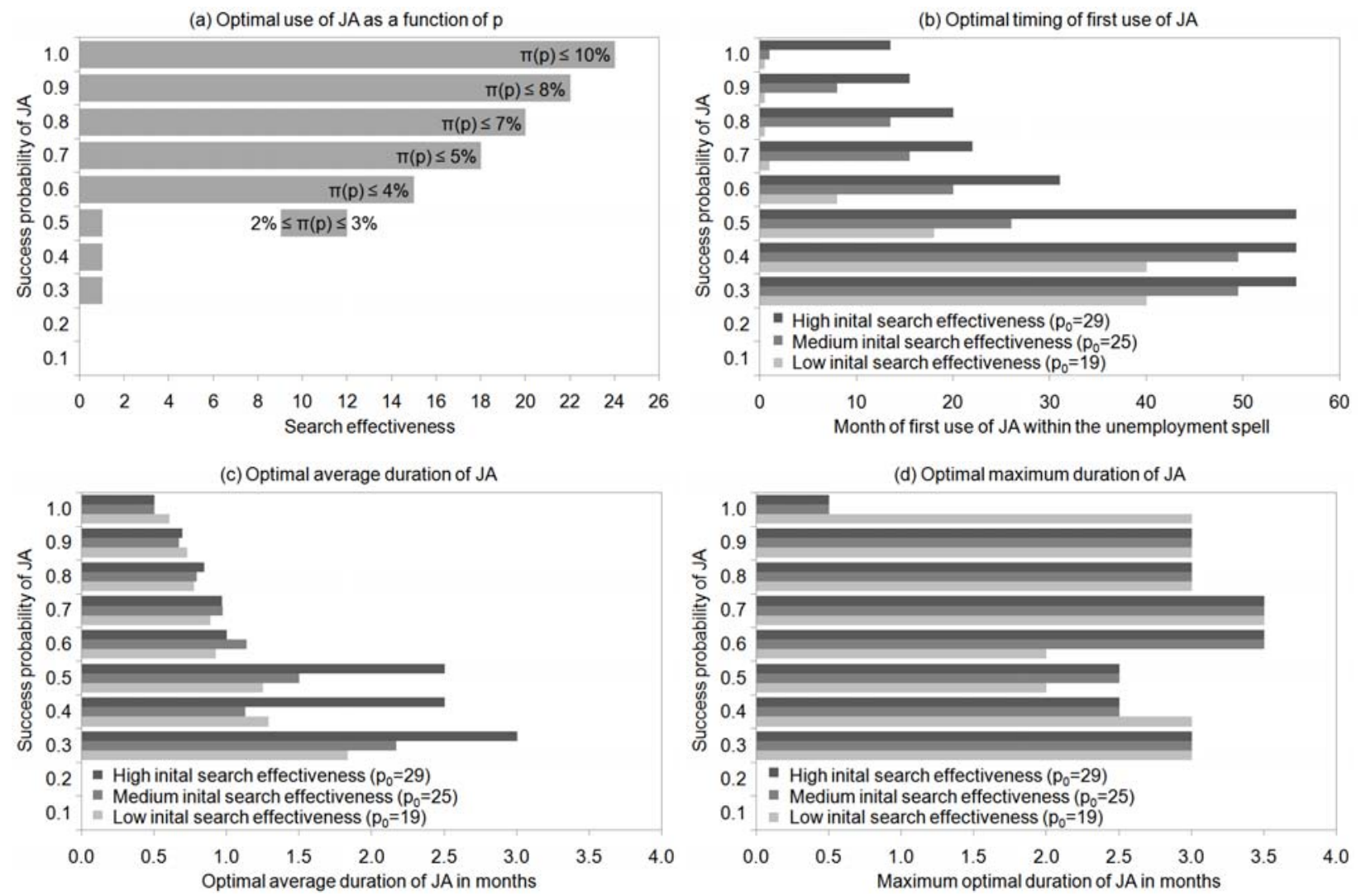

The results in panel (a) have direct implications for the optimal timing of JA within the unemployment spell and its optimal duration. Whenever initial search effectiveness, $p_{0}$, lies within the displayed range of $p$, JA should be used at the beginning of unemployment. Otherwise, it should only be used later in the unemployment spell once search effectiveness has deteriorated to a level within this range. Moreover, for $\theta \geq 0.6$, JA should be used until the the upper bound of the displayed range of $p$ is reached. Thereafter, UI will usually be optimal until $p$ depreciates below the threshold level that makes JA optimal once again. Essentially, after JA has increased $p$ to the threshold level, there will be multiple switches between UI and JA. Consequently, the combination of JA and UI completely crowds out the use of both SA and JM. The only case where JM would be optimal rather than UI is when promised utility is very high because incentivizing the worker would be too costly in this case (see Figure 2). In summary, JA can be used to prevent situations in which the returns to search are so low that it is no longer optimal to incentivize the worker and SA becomes optimal. In particular, JA can restore or maintain some minimum exit rate to employment 
that increases with the success probability of JA. As a result, it dominates both SA and JM, leaving UI as the optimal policy whenever JA is not optimal.

Panel (b) of Figure 6 shows the optimal timing of JA within the unemployment spell for three types of workers that represent the lower $(p=19)$, middle $(p=25)$, and upper third $(p=29)$ of the distribution of search effectiveness in the data. Corresponding to the range of values of $p$ displayed in panel (a) for which JA is optimal, JA should only be used relatively late in the unemployment spells when initial search effectiveness is high. The same holds for medium levels of $p_{0}$ as long as $\theta$ is below its maximum. For workers with low $p_{0}$, JA is optimal at the beginning of unemployment for $\theta \geq 0.8$, and it should start after one half-month of unemployment for $\theta=0.7$. At lower levels of $\theta$, JA should only be used with longer unemployment durations. Note, however, from Figure 5 that for $p_{0}=19$, the implied effect on the exit rate is still moderate even for $\theta \geq 0.8$, which suggests that under normal circumstances JA should be used at the beginning of unemployment for workers with relatively low search effectiveness. For all other workers JA, should only be used if they fail to find a job within reasonable time despite high expected initial exit rates. Note also that early use of JA is actually common practice in most existing WTW programs. However, in many countries, including Germany, JA is used quite extensively for all types of workers. In these countries the targeting of JA should be improved by focusing the use of JA on workers with relatively low predicted exit rates.

Panels (c) and (d) of Figure 6 show the optimal average and maximum durations of JA for the three representative types of workers. As is common in the majority of existing WTW programs, the optimal duration of JA is short. It rarely exceeds one month and never exceeds 3.5 months. The optimal duration depends on how long a worker stays at a particular level of $p$. On the one hand, this is determined by the success probability of JA: the higher $\theta$, the faster the boundary of $p$ from which JA will no longer be optimal is reached. Consequently, the optimal duration of JA decreases with increasing $\theta$. On the other hand, the depreciation probability of $p$ negatively affects how long workers stay at a given $p$.

The budget implications of the availability of effective JA depend on both the initial search effectiveness, $p_{0}$, and the success probability, $\theta$. Naturally, the budget savings are larger the larger $\theta$. Moreover, as costly SA without tax revenue is the dominant policy early in the unemployment spell when $p_{0}$ is small, and since JA can be used to prevent this, they are also higher the lower $p_{0}$. Consequently, for workers with low initial search effectiveness $\left(p_{0}=19\right)$, the additional budget 
savings relative to the optimal policy with SA, UI, and JM range from $2 \%$ to as much as $28 \%$ as $\theta$ varies from 0.3 to 1 . For workers with medium initial search effectiveness $\left(p_{0}=25\right)$, the savings are considerably smaller, ranging from below $1 \%$ to at most $4 \%$. The budget savings for a worker with high initial search effectiveness $\left(p_{0}=29\right)$ are negligible as they are below $1 \%$ for all values of $\theta$.

Figure 7: Varying the fixed cost of JA
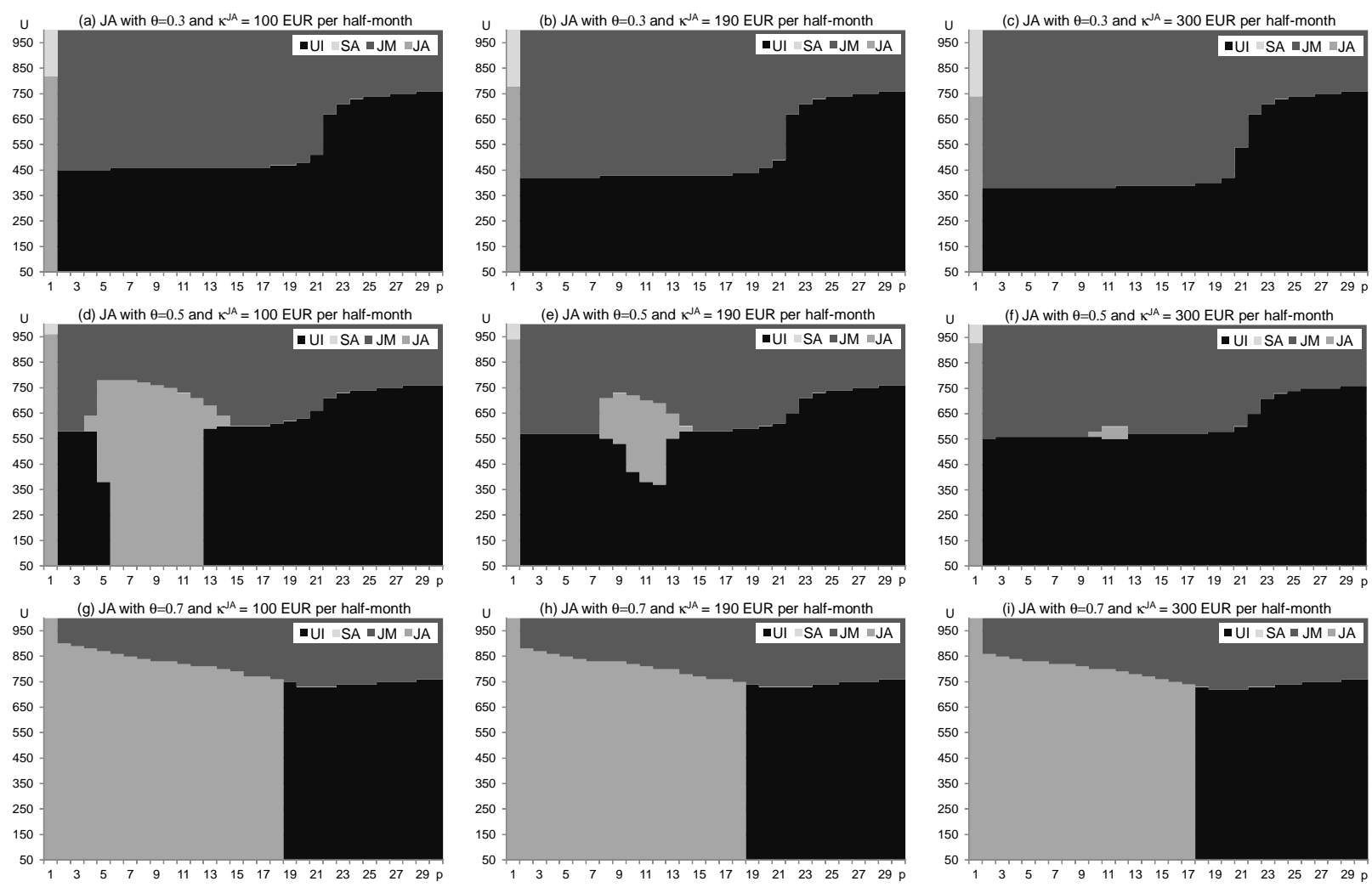

Note: At the right boundary of $p$ where the optimal policy switches from JA to UI, $V^{J A}$ and $V^{U I}$ are very close. Therefore, it can sometimes happen that there are multiple switches between JA and UI as $U$ increases for a given $p$. In such cases, the figures show UI up to the largest value of $U$ for which UI is optimal.

Figure 7 finally illustrates for selected values of $\theta$ how the optimal use of JA depends on the fixed $\operatorname{cost} \kappa^{J A}$. The baseline value is 190 EUR per half-month. The corresponding optimal policy choices in the $(U, p)$ space are displayed in panels (b), (e), and (h). In panels (a), (d), and (g) the fixed cost is reduced to 100 EUR per half-month, which implies equal fixed costs for JM and JA. In panels (c), (f), and (i), the fixed cost is increased to 300 EUR per half-month. The range of values of both $U$ and $p$ for which JA is optimal increases as the fixed cost decreases. Consequently, the use of UI also increases, while the use of JM decreases. However, the overall picture remains unchanged in the sense that JA is not optimal for $\theta \leq 0.2$, is only optimal for $p=1$ when $0.2<\theta \leq 0.4$, is optimal for both $p=1$ and intermediate values of $(U, p)$ at $\theta=0.5$, and is universally optimal up to some threshold values of $U$ and $p$ for $\theta \geq 0.6$. 


\subsubsection{Heterogeneity in reemployment wages}

So far, we have focused on the role of search effectiveness and the exit rate to employment. In this section, we want to study how ex-ante heterogeneity in reemployment wages and wage depreciation affect the optimal choice of policies. Besides the job finding probability, reemployment wages are the second main determinant of the returns to search. The higher the reemployment wage, the less costly it is to implement a certain utility level $U^{s}$ and hence to provide search incentives to the worker. ${ }^{27}$ Moreover, revenues from wage taxes are potentially higher or expenditures on wage subsidies lower.

Figure 8: Heterogeneity in reemployment wages
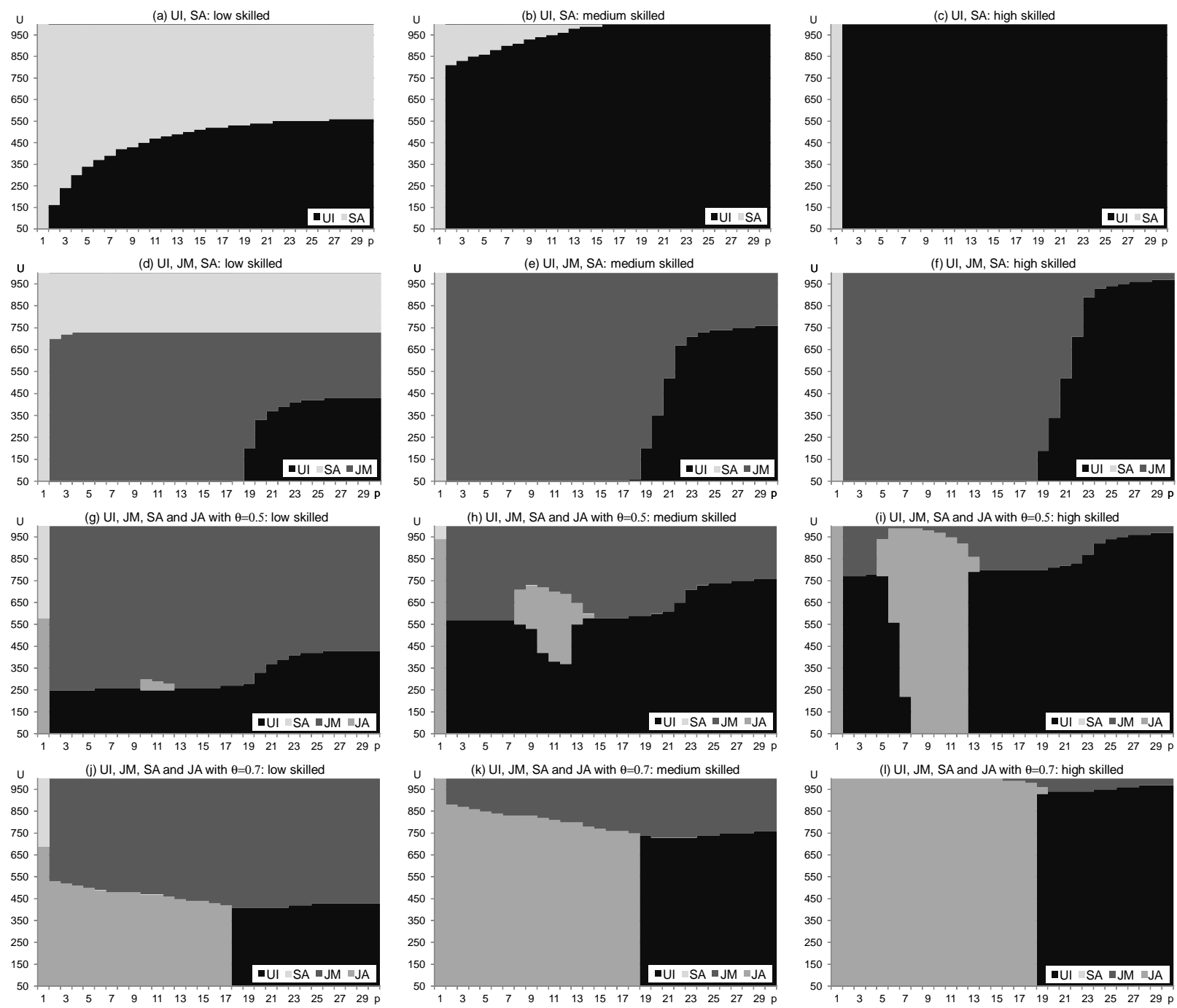

Note: Low/medium/high skilled: $w=880 / 1720 / 2680$ EUR per month. At the right boundary of $p$ where the optimal policy switches from JA to UI, $V^{J A}$ and $V^{U I}$ are very close. Therefore, it can sometimes happen that there are multiple switches between JA and UI as $U$ increases for a given $p$. In such cases, the figures show UI up to the largest value of $U$ for which UI is optimal.

$\overline{27}$ The level of $U^{s}$ is the return to search for the worker, and the utility spread $U^{s}-U^{f}$ determines the incentive cost for the planner. 
Figure 8 displays the optimal choice of policies in the $(U, p)$ space for, respectively, a low, medium, and high-skilled worker when different choice sets are available. The three types of workers are defined by the median wage in the lower, middle, and upper thirds of the wage distribution. As providing search incentives becomes more costly the lower $w$, the range of $U$ for which it is no longer optimal to incentivize the worker and SA is optimal increases considerably, even for high levels of $p$ (see panels (a)-(c)). In return, the use of both UI and, if available, JM is reduced (see panels (d)-(f)). Consequently, for any given level of initial promised utility, SA will be used earlier in the unemployment spell the lower $w$. The relative share of JM in the remaining periods before SA will increase, meaning that it will also be used earlier in the spell while the total use of UI will be reduced.

The optimality of JA also depends on reemployment wages. The reason is that the value of raising $p$ increases with $w$ : with the same increase in the exit rate, $\pi(p)$, a higher value of work can be obtained the larger $w$. Thus, the total returns to JA are larger for higher reemployment wages. One implication of this is that the minimum effectiveness required for JA to be used at all falls with increasing $w$. Moreover, as can be seen from panels (g)-(l), the range of values of $U$ and $p$ for which JA is optimal increases considerably with $w$ in terms of both $p$ and $U$. As discussed in Section 3.4.1, this also extends the use of UI and reduces the use of JM. Consequently, JA is more likely to be optimal the lower the search effectiveness, $p$, and hence the exit rate, $\pi(p)$, but the higher the reemployment wage, $w$.

Given these results, it is straightforward to derive what happens when there is not only ex-ante heterogeneity in reemployment wages but also wage depreciation during unemployment. With wage depreciation in addition to negative duration dependence in the exit rate to employment, both SA and JM will be used earlier in the unemployment spell, whereas the use of UI will be reduced compared with the case where wages remain constant. The importance of JA will diminish for two reasons. First, JA will be optimal in fewer cases. Second, it will lose its ability to prevent SA as the wage will eventually be so low that SA will already be optimal at very low levels of $U$ even at high levels of $p$ (see panel (a)). Thus, JA may delay the use of SA but cannot prevent it entirely when reemployment wages depreciate.

Having derived the optimal policy for different levels of the job finding probability and reemployment wages, it is interesting to discuss what may happen in countries like the US, which are 
characterized by much more flexible labor markets than countries like Germany. The most important difference is probably that the job finding probabilities are higher while wages are lower in the US than in Germany (see, e.g., the calibration by Pavoni and Violante (2007) for the US). Moreover, negative duration dependence in the exit rate to employment is probably smaller and wage depreciation larger in the US than in Germany. Given the same initial promised utility in both countries, the effects on the optimal use of SA, JM, and UI are ambiguous. Higher job finding rates and weaker duration dependence delay the use of JM and SA in favor of UI, while lower wages and higher wage depreciation imply the opposite. The implications for the use of JA are unambiguous, though, because both effects work in the same direction: the role of JA in optimal WTW programs in economies like the US will be much less prominent because of lower returns to JA, as the value of work is relatively low and falling rapidly during unemployment, while the job finding probabilities are high anyway. If we take into account that the US system is less generous than the German one, which implies lower initial promised utility, the differences will be smaller.

\subsubsection{Sensitivity to the preference parameters}

To check the sensitivity of the results with respect to the preference parameters of the model, we first vary the utility function of the worker. Rather than $\log$ utility, $u(c)=\frac{c^{1-\sigma}}{1-\sigma}$ is chosen with intertemporal elasticity of substitution once above and once below one $(\sigma \in\{0.8,1.2\}) .{ }^{28}$ Note that if $\sigma$ is below (above) one, the initial utility provided by the actual policy is higher (lower) than in the baseline case, because the same level of consumption implies higher (lower) utility. Moreover, for the same reason a given utility difference can be obtained with a smaller (larger) difference in benefits, implying that benefits must decline more slowly (faster) upon failure of the activity when the incentive constraint binds under UI or JA. However, it is found that the optimal sequence of policies remains almost unchanged. The only change is for $\sigma<1$ where JM and JA are used slightly earlier in the unemployment spell, i.e. for slightly higher $p$.

Second, the disutility of effort, $e$, is allowed to vary between work and search. The simulation is repeated for the case where the disutility of effort is assumed to be higher than the disutility of work. The higher value of $e=0.93$ rather than 0.84 in the baseline case is obtained when assuming that the time the worker would like to spend on search is only half of the time he would spend on work. Higher $e$ implies higher incentive costs during UI and JA, but also makes work relatively more

\footnotetext{
${ }^{28}$ The utility functions are normalized to match $u(1)=0$ as under log utility.
} 
attractive. In the simulation, these effects seem to offset each other, because the optimal choice of policies remains unchanged.

\subsubsection{How to implement the optimal policy}

An important issue is how the optimal policy could be implemented. As shown above, the optimal amount of benefits and sequence of policies depend on both the reemployment wage and the job finding probability, as well as on how they change with the unemployment duration. Consequently, implementing the optimal policy requires knowledge of the initial values $w_{0}$ and $\pi_{0}$ for each individual as well as their depreciation rates. The initial wage, $w_{0}$, is observed from the last employment spell and can be used to calculate the benefit replacement rates. For the wage depreciation rate and the exit rates to employment at any unemployment duration, a profiling approach would be required. The first step would be to estimate from historical data how individual characteristics affect $w_{t}$ and $\pi_{t}$ for any unemployment duration $t$. Based on the estimated coefficients, the predicted values should be calculated for each individual at the beginning of unemployment and should be updated whenever individual characteristics (like health or family situation) change over time.

Benefit claims should depend on the last wage and should take into account the unemployment risk. The reason is that the unemployment risk is highly correlated with the job finding probability once unemployed, and it will be more difficult to condition benefits on expected job finding rates. One way of accounting for unemployment risks would be to relate the amount of benefits to previous employment durations and unemployment experiences. Conditioning benefits on past wages and (un)employment experiences is actually common practice in most industrialized OECD countries. However, in contrast to existing policies, benefit claims should be defined in ex-ante expected value terms rather than as a fixed amount per period. Conditional on the predicted individual wage depreciation and job finding rates, they should then be distributed differently over time during unemployment for different types of workers. This approach is more likely to prevent that the discrimination in benefit levels across individuals and time required under the optimal policy beyond the heterogeneity in ex-ante expected value terms might be perceived as unjust. 


\section{Conclusion}

Job search assistance programs are one of the most widely used instruments for unemployed workers in OECD countries. This paper studies the role of such programs in optimal welfare-to-work programs based on an adapted version of the framework developed by Pavoni and Violante (2007). Besides endogenizing all major policy parameters and allowing for a dynamic environment, a key strength of the framework is that it takes into account the fact that there are alternative ways to use available funds to reach a given policy objective. As a consequence, cost-effectiveness is a necessary but not sufficient condition for job search assistance to be part of the optimal policy scheme. It has to be the least costly among all available policy instruments in a given period to be optimal in that period.

In contrast to Pavoni and Violante (2007), the adapted version of the framework that we propose takes into account the fact that certain worker characteristics like job search skills affect the job finding probability but not productivity in the job. This implies additional sources of ex-ante worker heterogeneity and differential duration dependence in the exit rates. As the optimal policy strongly depends on differences in the job finding probabilities, Pavoni and Violante (2007) neglect some important factors that may lead to different optimal decisions. Moreover, we show that introducing job search assistance has important implications for the dynamics of the model: in contrast to Pavoni and Violante (2007), depreciation in reemployment wages or negative duration dependence in the exit rates to employment are no longer necessary for policy transitions to occur during unemployment. If job search assistance is sufficiently effective and job finding probabilities are low, it may be optimal to use JA at the beginning of unemployment. As search effectiveness increases because of JA, it will eventually be sufficiently large for UI or JM to be optimal.

In the quantitative analysis the model is simulated for an economy that resembles West Germany in the period 2000-2002. One of the advantages of using West Germany is the availability of exceptionally rich and large administrative data that allow for most parameters of the model to be estimated nonparametrically for the same sample of interest, which is a large improvement over most existing calibrations of economic models. A comparison of the policy actually implemented in West Germany in the period 2000-2002 with the optimal scheme indicates a substantial inefficiency of the German system with respect to both benefit payments and the use of JA. Actual long-run benefits were too high and expenditures on JA were wasted as JA was ineffective in this period. 
We also show that there would have been considerable gains from implementing tight monitoring in West Germany, at least for workers with relatively low initial exit rates to employment.

Acknowledging the finding of the empirical literature that the effects of JA vary with the particular type of program as well as the time period and country considered, we analyze the optimal use of JA as a function of its ability to raise exit rates to employment. For the West German setting we find that the effect of JA on the exit rate to employment has to exceed $3 \%$. As this number is at the lower bound of the empirical estimates, JA will usually be part of optimal WTW programs. In line with existing policies, JA should mainly be used at the beginning of unemployment and for short durations. However, contrary to existing schemes, JA should be exclusively targeted at unemployed workers with low initial exit rates to employment. For all other workers, JA should only be used if they fail to find a job within reasonable time despite high expected initial exit rates. The budget savings from the availability of effective JA can be substantial, reaching as much as $30 \%$ for some types of workers compared with the optimal policy when JA is unavailable.

In economies without or only slow depreciation in reemployment wages, the main role of JA in optimal WTW programs is to prevent situations in which it is no longer optimal to incentivize the worker to provide positive search effort. In such situations, exit rates to employment and hence tax revenues would be zero, while expenditures on benefits would be high. JA is used to restore or maintain some minimum exit rate to employment which increases with the effectiveness of JA and with lower costs of JA. Because of this feature, JA dominates the use of monitoring: JM is an important instrument to reduce incentive costs when job finding probabilities deteriorate because this makes providing search incentives more costly, but, in contrast to JA, it cannot prevent exit rates from declining.

In economies with rapid depreciation in reemployment wages, JA plays a less prominent role. As the returns to JA decline with reemployment wages, JA will be optimal in fewer cases. Moreover, it can only delay but no longer prevent the deterioration of the returns to search because it cannot stop reemployment wages from declining. In this case, the results obtained by Spinnewijn (2009) and Wunsch $(2009 b)$ suggest, that a combination of job search assistance and training that increases human capital and thus reemployment wages would be required to keep the returns to search sufficiently high. 


\section{References}

Abbring, J., G. VAn Den Berg and J. VAn Ours (2005): The Effect of Unemployment Insurance Sanctions on the Transition Rate from Unemployment to Employment, Economic Journal, 115(505), pp. 602-630.

BA (2001-2005): Arbeitsstatistik - Jahreszahlen 2000, 2001, 2002, 2003, 2004, Amtliche Nachrichten der Bundesagentur für Arbeit, 49-53.

Blanchard, O. and J. Tirole (2008): The Joint Design of Unemployment Insurance and Employment Protection: A First Pass, Journal of the European Economic Association, 6(1), pp. 45-77.

Blundell, R., C. Meghir, M. Costa Dias and J. Van Reenen (2004): Evaluating the Employment Impact of a Mandatory Job Search Program, Journal of the European Economic Association, 2(4), p. $569-606$.

BMAS (2003): Statistisches Taschenbuch 2003: Arbeits- und Sozialstatistik. Bundesministerium für Arbeit und Soziales.

Boone, J., P. Fredriksson, B. Holmlund and J. Van Ours (2007): Optimal Unemployment Insurance with Monitoring and Sanctions, Economic Journal, 117(518), pp. 399-421.

Cahuc, P. and E. Lehmann (2000): Should Unemployment Benefits Decrease with the Unemployment Spell?, Journal of Public Economics, 77(1), pp. 135-153.

Cardullo, G. and B. Van der Linden (2007): Employment Subsidies and Substitutable Skills: An Equilibrium Matching Approach, Applied Economics Quarterly, 53(4), pp. 375-404.

Chari, V., L. Christiano and P. Kenhoe (1995): Policy Analysis in Business Cycle Models, in: T. Cooley (editor), Frontiers of Business Cycle Research, pp. 357-391, Princeton University Press, Princeton, New Jersey.

Coles, M. and A. Masters (2000): Retraining and Long-term Unemployment in a Model of Unlearning by Not Doing, European Economic Review, 44(9), pp. 1801-1822.

Coles, M. and E. Smith (1998): Marketplaces and Matching, International Economic Review, 39(1), pp. $239-254$.

Davidson, C. and S. Woodbury (1997): Optimal Unemployment Insurance, Journal of Public Economics, 64(3), pp. 359-387.

Dolton, P. and D. O'Neill (2002): The Long-Run Effects of Unemployment Monitoring and Work-Search Programs: Experimental Evidence from the United Kingdom, Journal of Labor Economics, 20(2), pp. 381-403.

FAY, R. (1996): Enhancing the Effectiveness of Active Labour Market Policies: Evidence from Program Evaluations in OECD Countries. Labour Market and Social Policy Occasional Papers, 18, OECD.

Flores-Lagunes, A., A. Gonzalez and T. C. Neumann (2007): Estimating the Effects of Length of Exposure to a Training Program: The Case of Job Corps. IZA Discussion Paper No. 2846.

Fredriksson, P. and B. Holmlund (2001): Optimal Unemployment Insurance in Search Equilibrium, Journal of Labor Economics, 19(2), p. 370-399.

Fredriksson, P. and B. Holmlund (2006): Optimal Unemployment Insurance Design: Time Limits, Monitoring, or Workfare?, International Tax and Public Finance, 13(5), pp. 565-585.

Gerfin, M. and M. Lechner (2002): Microeconometric Evaluation of the Active Labour Market Policy in Switzerland, Economic Journal, 112(482), pp. 854-893.

Heckman, J. (1979): Sample Selection Bias as a Specification Error, Econometrica, 47(1), pp. 153-162.

Heckman, J., R. LaLonde and J. Smith (1999): The Economics and Econometrics of Active Labor Market Programs, in: O. Ashenfelter and D. Card (editors), Handbook of Labour Economics, volume 3, pp. 1865-2097, North-Holland, Amsterdam. 
Hirano, K. and G. Imbens (2004): The Propensity Score with Continuous Treatments, in: A. Gelman and X.-L. Meng (editors), Applied Bayesian Modeling and Causal Inference from Incomplete-Data Perspectives, pp. 73-84, John Wiley and Sons, West Sussex.

Hopenhayn, H. and J. Nicolini (1997): Optimal Unemployment Insurance, Journal of Political Economy, 105(2), pp. 412-438.

Hopenhayn, H. and J. NiCOlini (2009): Optimal Unemployment Insurance and Employment History, Review of Economic Studies, 76(3), pp. 1049-1070.

Jespersen, S., J. Munch and L. Skipper (2008): Costs and Benefits of Danish Active Labour Market Programmes, Labour Economics, 15(5), pp. 859-884.

Kluve, J. and C. Schmidt (2002): Can Training and Employment Subsidies Combat European Unemployment?, Economic Policy, 35, pp. 411-448.

Lalive, R., J. VAn Ours and J. Zweimüller (2005): The Effect of Benefit Sanction on the Duration of Unemployment, Journal of the European Economic Association, 3(6), pp. 1386-1417.

Lechner, M. (2009): Sequential Causal Models for the Evaluation of Labor Market Programs, Journal of Business Economics and Statistics, 27, pp. 71-83.

Lechner, M. and R. Miquel (2010): Identification of the Effects of Dynamic Treatments by Sequential Conditional Idependence Assumptions, Empirical Economics. Forthcoming.

Lechner, M., R. Miquel and C. Wunsch (2010): Long-run Effects of Public Sector Sponsored Training in West Germany, Journal of the European Economic Association. Forthcoming.

Lechner, M. and S. Wiehler (2010): Does the Timing of Active Labour Market Programmes Matter?, Oxford Bulletin of Economics and Statistics. Forthcoming.

Lechner, M. and C. Wunsch (2009): Are Training Programs More Effective When Unemployment is High?, Journal of Labor Economics, 27(4), pp. 653-692.

Machin, S. and A. Manning (1999): The Causes and Consequences of Longterm Unemployment in Europe, in: O. Ashenfelter and D. Card (editors), Handbook of Labour Economics, volume 3, pp. 3085-3139, NorthHolland, Amsterdam.

Martin, J. and D. GrubB (2001): What Works and for Whom: A Review of OECD Countries' Experiences with Active Labour Market Policies, Swedish Economic Policy Review, 8, pp. 9-56.

OECD (2005): OECD Employment Outlook 2005. Chapter 4, Paris.

OECD (2007): OECD Employment Outlook 2007. Paris.

Pavoni, N. and G. Violante (2005): Optimal Welfare-to-Work Programs. Federal Reserve Bank of Minneapolis Discussion Paper 143.

Pavoni, N. and G. Violante (2007): Optimal Welfare-to-Work Programs, Review of Economic Studies, 74(1), pp. 283-318.

RiBi, E. (2009): Optimal Size and Intensity of Job Search Assistance Programs. Discussion Paper 2009-19, Department of Economics, University of St. Gallen.

Setty, O. (2009): Optimal Unemployment Insurance with Monitoring. New York University.

Shavell, S. and L. Weiss (1979): The Optimal Payment of Unemployment Insurance Benefits over Time, Journal of Political Economy, 87(6), pp. 1347-1362.

SiAnesi, B. (2004): An Evaluation of the Swedish System of Active Labour Market Programs in the 1990s, Review of Economics and Statistics, 86(1), pp. 133-155.

SiAnesi, B. (2007): Differential Effects of Swedish Active Labour Market Programs for Unemployed Adults during the 1990s. Revised version of IFS Working Paper W01/25. 
Spear, S. and S. Srivastava (1987): On Repeated Moral Hazard with Discounting, Review of Economic Studies, 54(4), pp. 599-617.

SpinnewiJn, J. (2009): Training and Search during Unemployment. Department of Economics, MIT.

Statistisches Bundesamt (2000-2002): Einnahmen und Ausgaben privater Haushalte, Wirtschaftsrechnungen. Fachserie 15, Reihe 1.

Thomsen, S. (2009): Job Search Assistance Programs in Europe: Evaluation Methods and Recent Empirical Findings. FEMM Working Paper No. 18, Otto-von-Guericke University Magdeburg.

Van den Berg, G., B. Van der Klaauw and J. Van Ours (2004): Punitive Sanctions and the Transition Rate from Welfare to Work, Journal of Labor Economics, 22(1), pp. 211-241.

Van Der Linden, B. (2003a): Unemployment Benefit Profile, Monitoring and Active Labor Market Policies. Document de travail no. 127 de la Chaire Hoover d'éthique économique et sociale, Université catholique de Louvain.

VAn Der Linden, B. (2003b): Unemployment Insurance and Training in an Equilibrium Matching Model with Heterogeneous Agents. IRES Discussion Paper No. 2003-01.

Van Ours, J. (2004): The Locking-in Effect of Subsidized Jobs, Journal of Comparative Economics, 32(1), pp. $37-52$.

Wang, C. and S. Williamson (1996): Unemployment Insurance With Moral Hazard in a Dynamic Economy, Carnegie Rochester Conference Series on Public Policy, 44, p. 1-41.

Wunsch, C. (2006): Labour Market Policy in Germany: Institutions, Instruments and Reforms since Unification. Revised version of Discussion Paper 2005-06, Department of Economics, University of St.Gallen.

Wunsch, C. (2007): Optimal Use of Labor Market Policies. Discussion Paper 2007-26, Department of Economics, University of St. Gallen.

Wunsch, C. (2009a): Optimal Monitoring and Sanctioning Systems. University of St. Gallen.

Wunsch, C. (2009b): To Train or Not to Train the Unemployed? University of St. Gallen.

Wunsch, C. and M. Lechner (2008): What Did All the Money Do? On the General Ineffectiveness of Recent West German Labour Market Programs, Kyklos, 61(1), pp. 134-174.

Zhно, R. (2000): The Optimal Unemployment Insurance Contract: Why a Replacement Ratio? University of Chicago. 


\section{Appendix: Proof of the proposition}

Pavoni and Violante (2007) show that with constant reemployment wages and in the absence of negative duration dependence in the exit rate to employment JM and SA are absorbing once they are optimal at some $(U, p)$. Thus, JA will never be used thereafter. Now consider the case where UI is optimal at $(U, p)$. Pavoni and Violante (2007) show that in the absence of JA, JM and SA will never be used thereafter, so the only choice is between JA and UI after UI. If $p$ remains constant during UI, it is sufficient to consider the dynamics with respect to $U$. The following Lemma establishes that whenever $w$ and $p$ do not depreciate and $\mathrm{UI}$ is optimal at $(U, p), V^{J A}$ is more negatively sloped than $V^{U I}$ if $\theta^{J A} \leq \pi(p)$ and, hence, JA will never be optimal in the next period.

Lemma: For every $(U, p)$ at which $U I$ is optimal and $\theta \leq \pi(p)$ we have that $V_{U}^{U I}(U, p) \geq$ $V_{U}^{J A}(U, p)$ if $V$ is submodular and $w$ and $p$ do not depreciate.

Proof of the Lemma: From the first-order and envelope conditions for UI and JA we have

$$
\begin{aligned}
& V_{U}^{U I}(U, p)=-\frac{1}{u^{\prime}\left(c_{U I}\right)}=\pi(p) V_{U}\left(U_{U I}^{s}, p\right)+(1-\pi(p)) V_{U}\left(U_{U I}^{f}, p\right) \\
& V_{U}^{J A}(U, p)=-\frac{1}{u^{\prime}\left(c_{J A}\right)}=\theta V_{U}\left(U_{J A}^{s}, p^{s}\right)+(1-\theta) V_{U}\left(U_{J A}^{f}, p\right),
\end{aligned}
$$

where $c^{U I}, U_{U I}^{f}$ and $U_{U I}^{s}$ are optimal consumption and continuation utilities under UI, and $c^{J A}$, $U_{J A}^{f}$ and $U_{J A}^{s}$ the respective values under JA. If $c^{U I} \leq c^{J A}$ the envelope condition and the concavity of $u$ imply the desired result. So consider the case where $\theta \leq \pi(p)$ but $c^{U I}>c^{J A}$. In this case we have that

$$
\begin{aligned}
-\frac{1}{u^{\prime}\left(c_{U I}\right)} & =\pi(p) V_{U}\left(U_{U I}^{s}, p\right)+(1-\pi(p)) V_{U}\left(U_{U I}^{f}, p\right) \\
& \leq \theta V_{U}\left(U_{U I}^{s}, p\right)+(1-\theta) V_{U}\left(U_{U I}^{f}, p\right) \\
& <\theta V_{U}\left(U_{J A}^{s}, p^{s}\right)+(1-\theta) V_{U}\left(U_{J A}^{f}, p\right)=-\frac{1}{u^{\prime}\left(c_{J A}\right)} \\
& \leq \theta V_{U}\left(U_{J A}^{s}, p\right)+(1-\theta) V_{U}\left(U_{J A}^{f}, p\right)
\end{aligned}
$$

Inequality (10) follows from $\theta \leq \pi(p), V_{U}<0$ and the concavity of $V$ while inequality (11) is implied by $c^{U I}>c^{J A}$. Inequality (12) follows from submodularity which implies that $V_{U}\left(U, p^{s}\right) \leq$ $V_{U}(U, p)$. Pavoni and Violante (2007) show that the IC constraint binds when UI is optimal, i.e. $U=u\left(c^{U I}\right)+\beta U_{U I}^{f}$. Satisfying the IC constraint under JA, i.e. $U \geq u\left(c^{J A}\right)+\beta U_{J A}^{f}$, with the same $U$ requires $U_{J A}^{f}>U_{U I}^{f}$ if $c^{U I}>c^{J A}$. Consequently, a necessary (but not sufficient) condition for

$$
\theta V_{U}\left(U_{U I}^{s}, p\right)+(1-\theta) V_{U}\left(U_{U I}^{f}, p\right) \leq \theta V_{U}\left(U_{J A}^{s}, p\right)+(1-\theta) V_{U}\left(U_{J A}^{f}, p\right)
$$

to be true is that $U_{J A}^{s}<U_{U I}^{s}$ since $V_{U}\left(U_{J A}^{f}, p\right) \leq V_{U}\left(U_{U I}^{f}, p\right)$ because $V$ is concave and $U_{J A}^{f}>U_{U I}^{f}$. However, the PK constraints and the IC constraint being binding under UI imply that

$$
U_{J A}^{s}-U_{J A}^{f} \geq \frac{e}{\beta \theta} \geq \frac{e}{\beta \pi(p)}=U_{U I}^{s}-U_{U I}^{f} \Leftrightarrow U_{J A}^{s}-U_{U I}^{s} \geq U_{J A}^{f}-U_{U I}^{f}>0
$$

which implies the contradiction that $U_{J A}^{s}>U_{U I}^{s}$. Thus, when $\theta \leq \pi(p), c^{U I}>c^{J A}$ cannot hold implying that $c^{U I} \leq c^{J A}$ must hold.

Since search effectiveness does not change during UI while promised utility falls, this completes the proof of the proposition. 